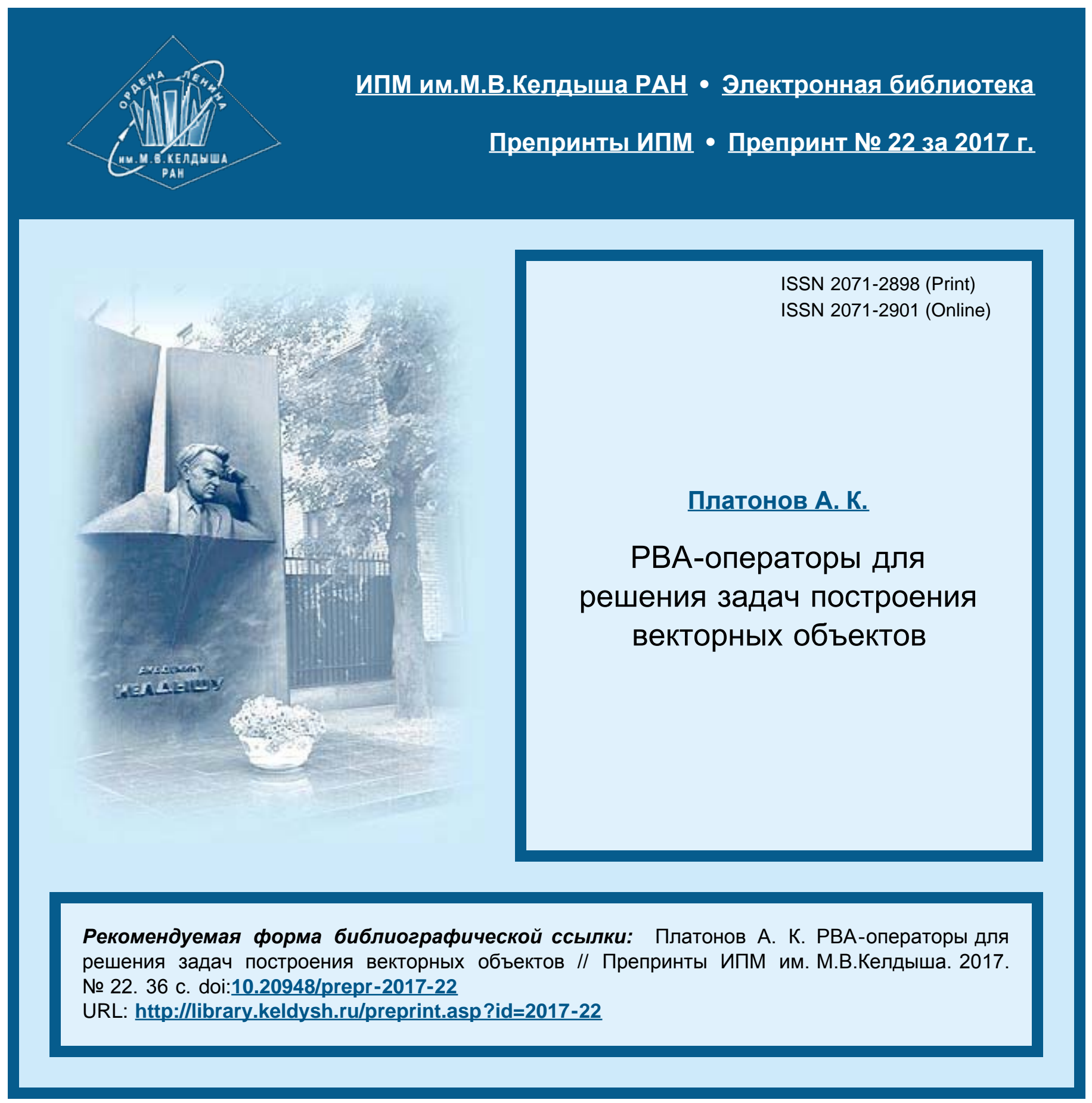




\author{
О рд н н а Л енина \\ ИНСТИТУТ ПРИКЛАДНОЙ МАТЕМАТИКИ \\ имени М.В. Келдыша \\ Р оссийской а ка дмиинаук
}

\begin{abstract}
А.К. Платонов
РВА-операторы для решения задач построения векторных объектов
\end{abstract}

Москва — 2017 
Платонов А.К.

PВА-операторы для решения задач построения векторных объектов Аннотация

Описан набор операторов векторной алгебры, расширяющий ее до алгебры геометрических операций, используемых как для решения задач ориентации и наведения направлений в пространстве, так и для задач позиционирования, прикосновения или пересечения векторных объектов в пространствах их систем координат.

Предлагаемые операторы векторных движений предназначены для численной реализации алгоритмов решения задач построения ориентации и позиционирования механизмов робототехники. Вместе с этим логика и содержание высказываний на языке множества операторов геометрических действий является эфффективным средством для внекоординатного описания взаимных положений объектов в пространстве.

Такое множество геометрических объектов и операций расширения векторной алгебры (PBA) полезно также и при формировании понятий в программах «машинного интеллекта».

Ключевые слова: системы ориентации космических аппаратов, кинематика манипуляторов, ориентация, позиционирование, прикосновение, совмещение, операторы геометрии

Работа выполнена при финансовой поддержке РФФИ (грант № 15-08-06431) и РНФ (грант № 16-19-10705).

\section{A.K. Platonov \\ VAE Operators for Solving Problems of Vector Objects Formation}

Paper describes a set of vector algebra operators extending its representation to what can be thought of as geometric operations. These operations are used solving orientation problems, problems of guidance and control definitions in a space as well as problems of positioning, contiguity and intersections of vector objects in their coordinate space.

Suggested operational set of vector motions helps with developing numerical algorithms aimed to be used for solving positional and orientational tasks in robotics. Furthermore, using the logic and semantics of clauses built with a language based on proposed set of geometric operators allows for efficient and generic non-coordinate descriptions of mutual special positions of objects in a space.

A set of geometric objects together with described system of operations which forms a Vector Algebra Extension (VAE) are also useful at semantic level descriptions in modern Al tasks.

Key words: guidance and control space systems, manipulator's kinematic, orientation, position making, touch, consisting, geometric operators.

The studies are carried out in RFBR grant № 15-08-06431

and RSF grant № 16-19-10705 


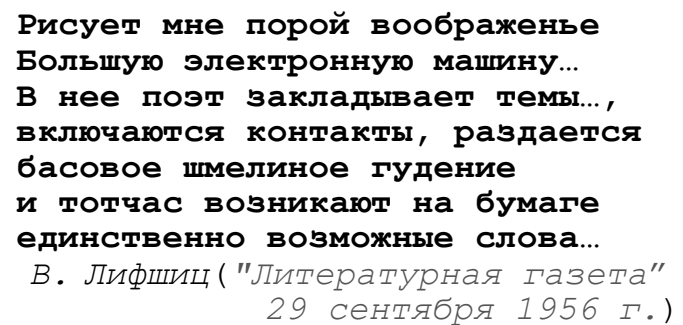

\section{ВВЕДЕНИЕ}

Известен факт, что Евклиду для получения единственно возможного решения в задачах геометрии были нужны лишь циркуль и линейка с палкой на песке (см., например, [1]). Это обстоятельство говорит не только о достаточности и полноте операторов видимого пересечения, совпадения и сравнения длин и углов, но, главное, и о том, что в большинстве случаев достаточно слов и нет необходимости в использовании чисел для решении задач геометрии как на плоскости, так и в пространстве (решения задач в пространстве сводятся к их решениям в трёх плоских чертежных проекциях).

Именно поэтому и в наше время возникает упрек геометров философу Декарту за не во всем правомерную замену образного геометрического мышления, нужного для решения задач, формализмом аналитической геометрии.

Можно утверждать, что Декарт заменил богатство видимости глазом и понимания мозгом изображаемых линейкой и циркулем плоских и пространственных геометрических объектов на видимость глазу и мозгу линейных изображений букв, чисел и специальных знаков арифметических и тригонометрических преобразований. Получаемые аналитически точные параметры метрики длин и углов описывают некоторые частные решения. Однако эти метрические параметры не нужны для общего решения каждой геометрической задачи, в которой нужно понять искомое геометрическое свойство, а не получить конкретное число.

Действительно, зачем, например, путаться внутри множества численных значений проекций на оси той или иной системы координат, выбор которой, к слову, отнюдь не однозначен? Ведь все геометрические преобразования движений и поворотов тел в топологическом, в римановом, в евклидовом или в проективном пространствах без привлечения чисел допускают некоординатные определения обстоятельств совмещения, совпадения, пересечения или прикосновения их объектов из-за присущего всем этим пространствам свойства инцидентности (сохранения внутренности) их элементов ${ }^{1}$.

\footnotetext{
${ }^{1}$ Строго говоря, для геометрических преобразований достаточно использования понятия аффинного пространства с линейно-выпуклыми комбинациями его «точек», реализуемых с помощью линейки или циркуля, - без определения ненужной нулевой «точки начала» этого пространства.
} 
Если принять, что основным методом решения геометрических задач является преобразование движения (т.е. преобразование с сохранением размеров объектов), то возникает желание описывать требуемые движения на языке целевых событий, не опускаясь на уровень метрики результата.

Конечно, метрические соотношения параметров геометрии необходимы и важны для практической реализации процессов проектирования и управления пространственными соотношениями реальных и виртуальных объектов. Но, записывая линейные строки формул аналитической геометрии, мы мысленно представляем себе их геометрическое содержание. И, более того, лишь после осмысления геометрии алгоритма решения мы отображаем это понимание в арифметические и тригонометрические операции покомпонентного описания реализации найденного векторного представления искомого решения. Поэтому алгоритмическое мышление вынужденно оказывается раздвоенным - и геометрическим, и координатным одновременно.

Однако теперь - в эпоху вычислительных машин - нет необходимости в задачах построения ориентации и позиционирования использовать метрику расстояний и тригонометрию углов в подробностях их координатного описания. Намного проще и удобнее, сформулировав операторно-геометрическое описание требуемых движений, получить затем нужное координатное представление своего алгоритма с помощью отдельной программной системы.

С целью реализации такой техники построения движений механизмов машин в ИПМ им. М.В. Келдыша была создана и успешно применялась, начиная с 1959 г., описываемая ниже библиотечная система геометрических операций расширения векторной алгебры (РВА). Эта система обеспечивала решения сначала задач космической техники, а затем (после потребовавшегося расширения состава операторов) и робототехники.

Система PBА позволяет программисту, сформулировав на языке геометрических понятий алгоритмы решения кинематических задач ориентации и позиционирования механических объектов, автоматически построить и реализовать требуемые движения, не вникая, как правило, в их координатнометрическое содержание.

Как показал последующий опыт, такая техника хорошо приспособлена также и к разработке средств визуального программирования для решения и исследования задач кинематики механизмов методами машинной графики.

Логика и содержание высказываний на языке операторов PBA о построении нужных геометрических действий является весьма эффективным средством для формирования семантики некоординатного описания взаимного положения и движения объектов в пространстве. Поэтому описываемая ниже система РВА 
приносит также пользу и для организации понятийного уровня в современных программных реализациях «машинного интеллекта».

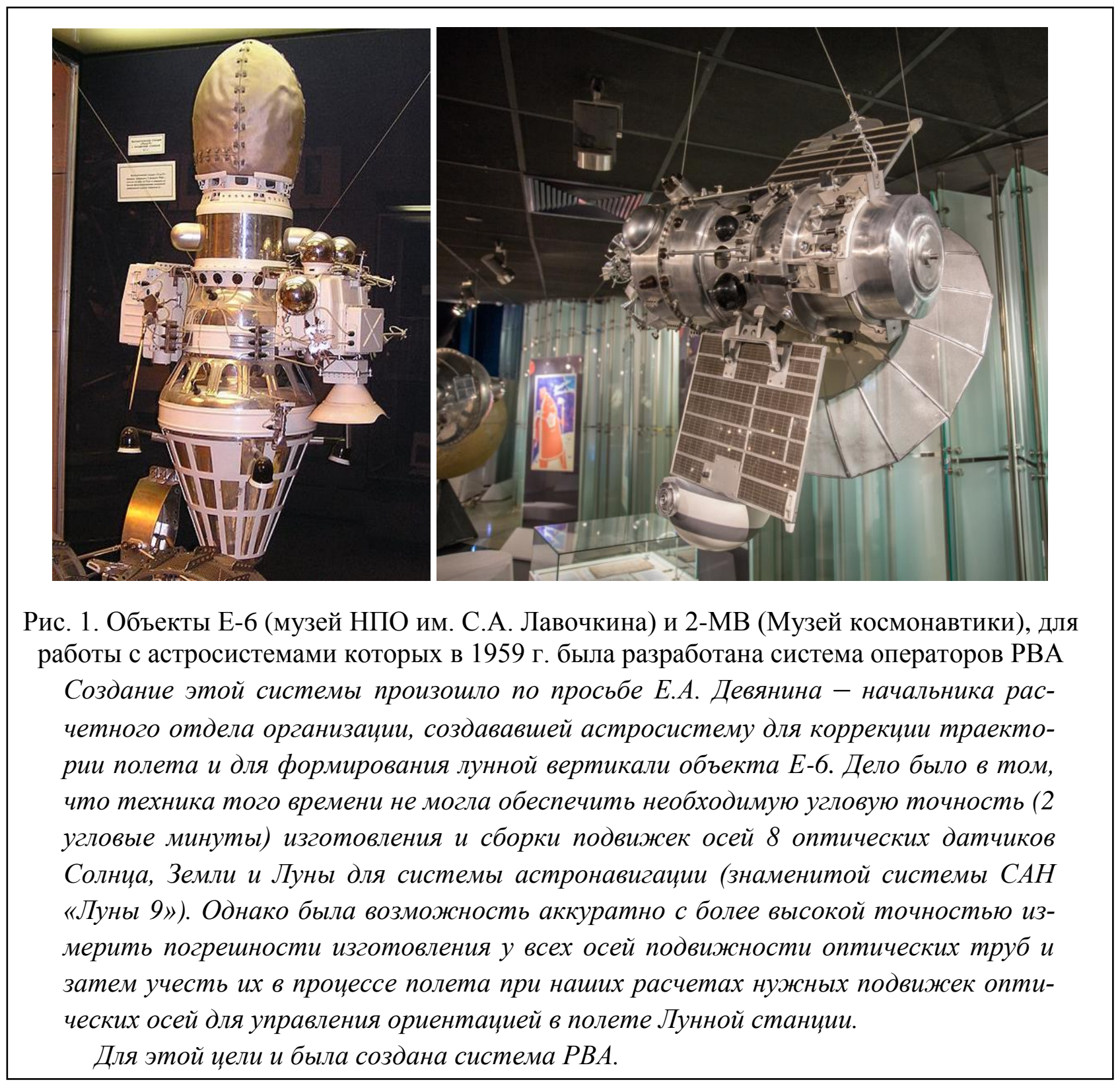

Предлагаемое ниже описание состава и содержания операторов для решения задач построения векторных объектов и их движений базируется на опыте лекций автора для студентов пятого года обучения на факультете управления и прикладной математики Московского физико-технического института. Здесь этот текст значительно расширен описанием возникающих обстоятельств построения движений роботов и новыми операторами, потребность в которых возникла для работы с пространственными (с перекрещиванием осей) кинематическими схемами манипуляторов.

Автор выражает искреннюю благодарность Ю.Ф. Голубеву, прочитавшему текст и сделавшего ряд ценных замечаний к его содержанию. 


\section{ГЕОМЕТРИЧЕСКОЕ МЫШЛЕНИЕ И КООРДИНАТНАЯ РЕАЛИЗАЦИЯ}

Геометрический подход к описанию алгоритмов построения движений тел в пространстве имеет большую историю [1-21, 23, 24, 27, 28], и он широко используется и в настоящее время. Более того, подобные системы уже реализованы во многих коммерческих системах. Например, их можно увидеть в современной технологии построения графических изображений для компьютерных игр [26] или в сфере компьютерного моделирования робототехнических систем [28]. Однако такие коммерческие системы весьма дороги и, что наиболее существенно, они, увы, закрыты для их необходимого алгоритмического развития, часто диктуемого решаемой задачей. И, конечно, они достаточно громоздки для непосредственного использования в бортовых системах управления.

В данной работе основное внимание уделяется понятийному описанию геометрических («внекоординатных») средств и методов построения моделей и алгоритмов, развитому для формирования процессов ориентации управляемых объектов в космическом пространстве и позиционирования механизмов робототехники в их операционном пространстве.

Приходится признать, что для каждого нового космического аппарата, как и нового робота, бортовые алгоритмы и программы управления ориентацией и/или позиционированием их механизмов «специально и во связи» практически пишутся заново. И, как показывает опыт, развитые средства РВА наиболее подходят для создания бортового программного обеспечения систем управления движением в силу их простоты и достаточности.

Отметим, что полезность описываемого ниже набора операторов РВА заключается не только в том, что можно дешевыми средствами быстро запрограммировать и решить прямую или обратную кинематическую задачу для любой новой кинематической схемы. Наибольшая польза РВА заключается в формировании геометрически-операционного способа мышления для быстрого построения алгоритмов решения сложных кинематических задач при создании математических и программных моделей управления движением агрегатов механики с их сложными многоосевыми кинематическими схемами. Представляется несомненным, что развитое в формализме операторов РВА «кинематическое мышление» алгоритмиста-программиста в понятиях операторов PBA обеспечивает быстрое понимание пути решения двигательных задач мехатроники - с возможной их реализацией или средствами РВА, или любыми иными имеющимися и привычными технологическими средствами программирования систем управления механизмами.

Из современных отечественных работ содержательно наиболее близки к данной работе геометрические методы, развитые на кафедре Инженерной гео- 
метрии и САПР профессором Ф.Н. Притыкиным из Омского государственного технического университета (ОмГТУ). Использование геометрических моделей многомерного операционного пространства тут тоже позволило разработать методы точного расчета векторов обобщенных скоростей элементов механизмов с учетом имеющихся конструктивных и операционных ограничений их движению. Большое внимание в этих работах уделено и проблеме построения безопасных движений манипулятора вблизи внешних тел $[7,15]$.

Заметим, что эта же проблема построения движений манипулятора среди препятствий была рассмотрена и очень удачно аппаратно решена В.П. Носковым в ранней работе Таганрогского политехнического института [9].

При программном решении геометрических задач ориентации и позиционирования тел или механизмов желание избавиться от покомпонентного координатного описания алгоритмов не позволяет, тем не менее, полностью избавиться от векторных описаний объектов предметного пространства. Иными словами, нельзя при описании геометрических объектов полностью отказаться от семантики векторов: в вычислительном плане вектор должен всегда иметь конкретное покомпонентное координатное описание. Дело в том, что компилятору в виде объектов реализации программных вычислений нужны и сообщаются именно численные значения компонентов векторов.

С другой стороны, для проектанта и программиста векторные объекты очень удобны именно тем, что они существуют сами по себе - вне выбранных осей систем координат и их покомпонентных описаний. В этом и было достижение великих ученых XIX в. - Ф. Гаусса, У. Р. Гамильтона, Дж. К. Максвелла, Дж. У. Гиббса и О. Хэвисайда, сформировавших понятие вектора, как понятие геометрического объекта, которое нашло новое применение в тех задачах физики и механики, где раньше господствовали комплексные числа и кватернионы.

Итак, векторы позволяют осмысливать задачу понятийно-геометрически, a реализовывать - покомпонентно. Поэтому векторно-операторный язык является объективно необходимым верхним уровнем кода в многоуровневой системе кодов программных описаний типа:

«Понятия» - «Алгоритмы» - «Реализация».

В этой ситуации наилучшим способом подняться выше покомпонентного описания нужных алгоритмов является распространение векторных описаний на описание не только отдельных векторов, но и крупных геометрических объектов и операций с ними. Но это приводит к необходимости расширения состава обычных операций векторной алгебры до системы векторных преобразований с учетом специфики устройства и движений сложных механизмов и объектов предметного пространства решаемых задач. 
Что представляют собой простейшие геометрические объекты?

В геометрии Евклида классами геометрических объектов и их метрическими параметрами, как известно, являются:

1. точка, прямая линия, отрезок прямой линии, вершина;

2. окружность, ее центр, радиус, диаметр, дуга, хорда, ее касательная;

3. круг, сектор круга, сегмент круга, центр, радиус, площадь, нормаль;

4. эллипс, его фокусы, его большая и его малая полуоси, его перицентр и его апоцентр, его касательные;

5. парабола, ее фокус, ее ось, ее перицентр, ее директрисы, ее касательные;

6. гипербола, ее фокус, ее перицентр, асимптоты, касательные, ее большая и малая полуоси;

7. видимый угол (степень несовпадения пересекающихся прямых линий), вершина угла, стороны угла, острый угол, прямой угол, тупой угол, биссектриса;

8. треугольник (можно подумать, что именно здесь появляется число число сторон геометрического объекта, однако это число не случайно заменено его именем: тут нет метрики, а есть лишь перечисление), углы, стороны, вершины, высоты, медианы, площадь, периметр;

9. четырехугольник, трапеция, параллелограмм, ромб, прямоугольник, квадрат, углы, диагонали, вершины, стороны, середины сторон и диагоналей, площадь, периметр;

10. четырехвершинник, углы, диагонали, вершины, стороны, середины сторон и диагоналей, площадь, периметр;

11. многоугольник, правильный многоугольник, вершины и их число, углы, диагонали, стороны, середины сторон и диагоналей, площадь, периметр;

12. пирамида, призма, тетраэдр, октаэдр, многогранник, число вершин, вершины, ребра, грани, диагонали, площади сторон, объем;

13. цилиндр, конус, образующая, вершина, основание, высота, боковая площадь, площадь основания(-й);

14. сфера, шар, эллипсоид, гиперболоид, параболоид, радиус, полуоси, площадь, объем;

15. тор, радиусы, площадь, объем.

Такие базовые объекты, их ориентация и позиция хорошо описываются векторами и совокупностью векторов в системе операторов РВА. Это позволяет в приложениях их определять и использовать как векторные объекты определенного типа - с присущими им поименованными свойствами и специальными методами преобразования численных параметров этих свойств. 


\section{ВИДЫ ИСПОЛЬЗУЕМЫХ ДАННЫХ}

Состав и имена данных и геометрических объектов системы PBA, их типы данных и семантика их взаимных видо-родовых отношений показаны в Таблицах 1 - 5. Параметры Таблиц 1 и 2 достаточно очевидны, но нужно подчеркнуть, что при формировании требуемых движений в задачах ориентации или позиционирования объектов в физическом пространстве обычные понятия «направления» или «величины» совмещаемых векторов используются по отдельности, как два содержательно по-разному необходимых понятия при описании алгоритмов решения этих задач. В связи с этим в таблицах возникают различные отдельные имена переменных одинакового типа данных.

Таблица 1

Базовые переменные

\begin{tabular}{|c|c|c|c|}
\hline Объект РВА & Имя, свойство & Семантика & Тип \\
\hline Имя объекта & NAME, $\forall$ лат.симв. & Его идентификатор & string \\
\hline Константа & С, $\forall$ число & Известная величина & const \\
\hline Число элементов & $\mathrm{N}$ & Управление перебором & int \\
\hline Радиус, длина, размер & $\mathrm{R}, \mathrm{L}, \mathrm{l}, \mathrm{NAME}$ & Метрика свойств объекта & double \\
\hline Расстояние & dist, $\mathrm{S}$ & Мера дальности и пути & double \\
\hline Направляющий косинус & $0 . \geq \cos _{-} \leq 1.0$ & Компоненты орта & double \\
\hline Угол & $\forall$ греческий символ & Мера отличия направлений & double \\
\hline Конечный поворот & $\mathrm{H}=\{\mathrm{E}(\mathrm{Ocь}), \mathrm{U}(У г о л) ~\}$ & Построение поворота & OsUg \\
\hline Перечень Q & $\alpha \beta$ үлли Э४ү или $\mathrm{H}_{1} \mathrm{H}_{2} \mathrm{H}_{3}$ & Построение направлений & double \\
\hline
\end{tabular}

Важно почеркнуть, что в задачах позиционирования в большинстве случаев содержание совмещаемых векторных объектов описывается не свободными векторами, а двойными векторами, связанными с отрезками прямых и с их положением в физическом пространстве решаемой задачи. Такие "D6data"векторы (они ниже кратко именуются «позиционируемыми векторами») обрабатываются как связанная пара векторов.

Таблица 2 Векторные переменные

\begin{tabular}{|c|c|c|c|}
\hline Объект & Имя типа объекта & Реализация & Tuп \\
\hline 3D-вектор & D3V или D3Data & Структура 3 вещ. чисел & vector \\
\hline 5D-вектор & D5V или D5Data & Структура 5 вещ. чисел & D5data \\
\hline 6D-вектор & D6V или D6Data & Структура 6 вещ. чисел & D6data \\
\hline Позиция отрезка, плоскости & $\{\mathbf{b}, \mathbf{b} e\}$ или $\{\mathbf{b}, \mathbf{N}\}$ & Структура 2 векторов & PozV \\
\hline 3 вектора отрезка, плоскости & $\{\mathrm{b}, \mathrm{e}, \mathrm{be})$ или $\{\mathbf{V} 1, \mathrm{~V} \mathbf{2}, \mathrm{V} \mathbf{3}\}$ & Структура 3 векторов & M3V \\
\hline Единичный 3D вектор & EV $=$ ort__=dir_ & 3Dвектор $\{\cos \mathrm{X}, \cos \mathrm{Y}, \cos \mathrm{Z}\}$ & vector \\
\hline Орты репера векторов & SMort & Структура 3 EV & M3ort \\
\hline 6 векторов или D6-RVрепер & S6v или SCoordVelosity & Структура 6 D3V & S6D3V \\
\hline N векторов объекта РВА & NVOb & Структура $\{\mathrm{N}, \mathrm{D} 3 \mathrm{~V} 1 . . \mathrm{D} 3 \mathrm{VN}\}$ & vob \\
\hline
\end{tabular}

B системе PBA содержатся обычные операции алгебры 3D- и Poz- векторов (в сложении и вычитании Poz-векторов отдельно суммируются обе их части): 
Таблица 3 Векторные операции 3D-свободных и Poz- векторов

\begin{tabular}{|c|c|c|}
\hline Оператор & \multicolumn{2}{|l|}{ Описание; ответ } \\
\hline Пересылка вектора & vector $\operatorname{VeV}($ vector $\mathbf{A})$; & \\
\hline Инверсия вектора & vector VInv(vector $\mathbf{A})$; & \\
\hline Модуль вектора & double VMod(vector $\mathbf{A}) ; \quad \mathbf{B}=|\mathbf{A}|$ & \\
\hline Единичный вектор & vector VEd(vector $\mathbf{A})$ & \\
\hline Модуль и длина вектора & \multicolumn{2}{|c|}{ vector VEd_M(vector $\mathbf{A}$, double*modA $) ; \mathbf{R}=\operatorname{dir} \mathbf{A}, \bmod A=\mid \mathbf{A}$} \\
\hline Умножение вектора на скаляр & \multicolumn{2}{|c|}{ vector VuS(vector $\mathbf{A}$, double $S) ; \quad$ B $=\mathbf{A} \cdot S$} \\
\hline Сложение векторов & \multicolumn{2}{|c|}{ vector $\mathrm{VpS}($ vector $\mathbf{A}$, vector $\mathbf{B}) ; \quad \mathrm{C}=\mathbf{A}+\mathbf{B}$} \\
\hline Вычитание векторов & \multicolumn{2}{|c|}{ vector VmS(vector $\mathbf{A}$, vector $\mathbf{B}) ; \quad \mathbf{C}=\mathbf{A}-\mathbf{B}$} \\
\hline Сумма и разность Poz-векторов & \multicolumn{2}{|c|}{ PozV PVp(или m)PV(PozV PA, PozV PB) C=A \pm B } \\
\hline Скалярное произведение & \multicolumn{2}{|c|}{ double VscalV(vector $\mathbf{A}$, vector $\mathbf{B}) ; \quad \mathrm{S}=(\mathbf{A}, \mathbf{B})$} \\
\hline Векторное произведение & \multicolumn{2}{|c|}{ vector VvectV(vector $\mathbf{A}$, vector $\mathbf{B}) ; \quad C=[\mathbf{A} \times \mathbf{B}]$} \\
\hline
\end{tabular}

В РВА включены 5 логических векторных операций, показанных в Таблице 4: Таблица 4 Логические 3D-векторные операции

\begin{tabular}{|l|l|}
\hline \multicolumn{1}{|c|}{ Оператор } & \multicolumn{1}{c|}{ Описание, ответ } \\
\hline \hline Коллинеарность двух векторов & int=IfVKollV(vector $\mathbf{A}$, vector $\mathbf{B}) ; \Rightarrow 0$ - нет, 1 - да \\
\hline Компланарность трёх векторов & int=IfVKompV(vector $\mathbf{A}$, vector $\mathbf{B}) ; \Rightarrow 0$ - нет, 1 - да \\
\hline$\varepsilon$-совпадение векторов $\left(\varepsilon=10^{-7}\right)$ & int IfVeV(vector $\mathbf{A}$, vector $\mathbf{B}) ; \quad \Rightarrow 0$ - нет, 1 - да \\
\hline Строгое равенство векторов & int IfVeqV(vector $\mathbf{A}$, vector $\mathbf{B}) ; \quad \Rightarrow 0$ - нет, 1 - да \\
\hline Неравенство модулей векторов & int IfVneqV(vector $\mathbf{A}$, vector $\mathbf{B}) ;|\mathbf{A}| \neq|\mathbf{B}| \Rightarrow 1$ - да, или 0 \\
\hline
\end{tabular}

Системы координат принадлежат специальному типу векторноматричных объектов. Типы этого объекта объединяют в одной SystCoord структуре три единичных вектора направляющих косинусов ортов рассматриваемой системы координат в осях внешней системы координат (см. табл. 5). Заметим, что в системе РВА все матрицы преобразований хранятся и операции над ними выполняются в скалярных произведениях векторов их строк или столбцов.

Таблица 5 Позиционные 9D-объекты типа «Система координат»

\begin{tabular}{|l|c|l|l||}
\hline \hline \multicolumn{1}{|c|}{ Объект } & Имя & \multicolumn{1}{|c|}{ Реализация } & \multicolumn{1}{c|}{ Tuп } \\
\hline \hline Базовая сист. координат & BSK & SK вида $\{1,0,0 ; 0,1,0 ; 0,0,1\}$ & SystKoord \\
\hline Объектная сист. координат & OSK & Струк-ра & SystKoord \\
\hline Вертикальная сист. коорд. & VSK & OSK с ortX, ortY $\uparrow$, ortZ & SystKoord \\
\hline Горизонтальная сист. коорд. & GSK & OSK с ortX, ortY, ortZ $\uparrow$ & SystKoord \\
\hline Присоединенная сист. коорд. & PSK & Структура \{2 D3V, SKort, 4 D3V & PSK \\
\hline Репер трех векторов в ПСК & R3V & Структура \{REort, PSK, 3 D3V & reap \\
\hline
\end{tabular}

Упомянутые в таблице 5 понятия Базовая и Объектная системы координат отличают систему координат исходного пространства задач от внутренних для них систем координат объектов этого пространства. 


\section{ПРИСОЕДИНЁННАЯ СИСТЕМА КООРДИНАТ И ОРТОГОНАЛИЗАЦИЯ ВЕКТОРОВ}

Особое значение среди систем координат системы объектов и операторов системы РВА имеют присоединенная система координат и репер трех векторов в присоединенной системе координат, упомянутые в последних строках таблицы 5. Понимание и описание содержания многих операторов РВА в этих системах координат значительно упрощается.

Отметим важное обстоятельство: в задачах определения ориентации и позиционирования системы координат достаточно определить требуемую позицию только двух любых связанных с ней векторов. Это обстоятельство приводит к семантической важности операторов работы с двумя связанными векторами при построении требуемого положения рассматриваемого объекта в его внешнем пространстве.

В системе РВА основным оператором для работы с двумя векторами является оператор $\operatorname{PSK}(\mathbf{A}, \mathbf{B})$. Оператор $\operatorname{PSK}(\mathbf{A}, \mathbf{B})$ - это оператор построения в исходной системе координат особой $3 \mathrm{D}-$ системы координат, названной «Присоединённой системой координат», определённым образом связанной с плоскостью и направлением двух заданных 3D-векторов. Оси этой встроенной в векторные понятия РВА системы координат определяют специальное векторное пространство, связанное с любой парой $\mathbf{A}$ и $\mathbf{B}$ неколлинеарных векторов.

По определению присоединённой системы координат (далее - ПСК) её ось $\mathbf{X}_{\text {пск }}^{0}$ направлена вдоль первого заданного вектора (A), ось $\mathbf{Y}_{\text {пск }}^{0}$ - ортогонально вектору $\mathbf{A}$ в полуплоскости $(\mathbf{A}, \mathbf{B})$, содержащей вектор $\mathbf{B}$, а ось $\mathbf{Z}_{\text {пск }}^{0}-$ по направлению векторного произведения $\mathbf{Z}_{\text {пск }}^{0}=[\mathbf{A} \times \mathbf{B}]^{0}$. Тогда :

$$
\mathbf{X}_{\text {Пск }}^{0}=\mathbf{A}^{0}, \quad \mathbf{Y}_{\text {Пск }}^{0}=\left[\mathbf{Z}_{\text {пСК }}^{0} \times \mathbf{A}\right]^{0} .
$$

Построенная с помощью оператора PSK присоединенная система координат, показанная ниже на рис. 2 и 3 , является эффективным инструментальным средством алгоритмов системы РВА. Она широко используется во многих операторах PBA. Семантически удобным свойством ПСК является возможность выполнять все требуемые векторные операции в нотации имен векторов присоединенного пространства, сохраняя при этом их компонентное представление в осях внешнего пространства исходной пары векторов.

Иными словами, представление исходных векторов в понятиях векторов ПСК позволяет пользоваться последними стандартно и независимо от выбора исходной системы координат, выполняя в осях ПСК все нужные преобразования - операции ортогонализации или совмещения векторов, операции определения углов между ними и операции градиентных (векторных) проекций. 


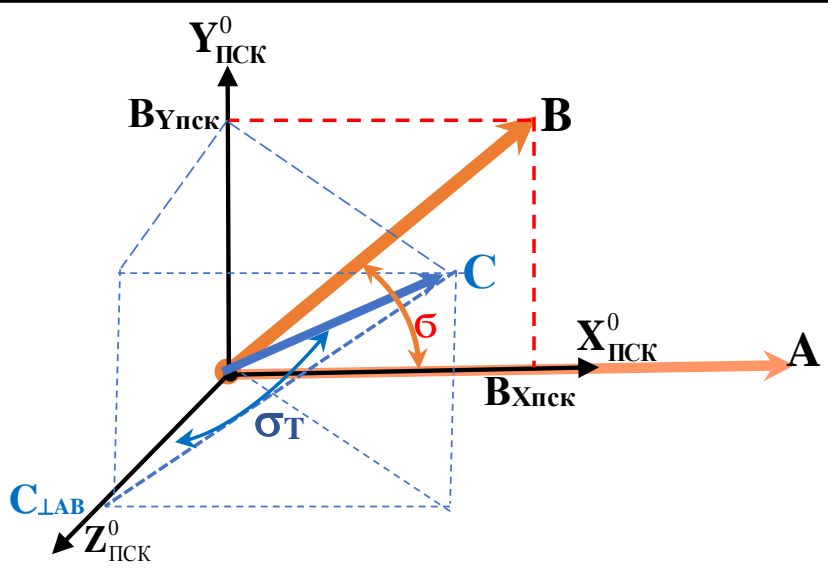

Рис.2. Репер трех градиентов и присоединенная к паре векторов система координат

Известное или требуемое положение пары векторов $\mathbf{A}$ и $\mathbf{B}$, фиксированное условиями задач ориентациии и позициионирования, играет роль начальных условий для многих задач. Показанная на рисунке связанная с такими векторами система координат позволяет универсальным способом формировать алгоритмы ремения этих задач, описывая относительно системной пары векторов свойства и движения других объектов (например, некоторого третьего вектора $\mathbf{C}$ ).

Если же системная пара векторов А и В является заданной парой векторов градиентов двух каких-либо скалярных переменных (строками матрицы линейной зависимости этих двух переменных $\xi$ и $\eta$ от компонент векторов исходного трехмерного пространства X, Y и Z), то система координат, присоединенная к векторам градиентов, определяет в исходном трехмерном пространстве положение и ориентацию их «оптимальной» плоскости $(\mathbf{A , B})$ в задаче формирования нужных значений выделенных переменных минимальными по величине векторами исходного пространства. Оптимальная плоскость определена ее нормалью («нуль-направлением») $\mathbf{Z}_{\text {пск }}^{0}$ Векторы вдоль этого направления не изменяют величины

Если некий третий вектор $\boldsymbol{C}$ тоже есть градиент зависимости еще одной третьей переменной $\zeta$ от векторов исходного пространства, то специальную роль играет его проекция на ось $\mathbf{Z}_{\text {пск }}^{0}$ присоединенной системы координат. Такая проекция определяет направление и величину условного градиента $C_{\mathrm{AB} \perp}$ для третьей переменной при условии ее изменения без изменения фиксированных значений величин $\xi$ и .

Построение ПСК активно используется при решении задач наведения на цель космических приборов, мобильных роботов и роботов-манипуляторов $[3,11,14]$.

Примерами двух системных векторов могут служить оси двух гироскопов в связанной системе координат космического аппарата, две оси «кисти» схвата манипулятора в системе координат основания манипулятора или вертикальная и продольная оси корпуса беспилотника, требуемое положение которых задано в мировой системе координат. В каждом из этих случаев присоединенная к упомянутым векторам система координат обладает полнотой описания действий в связанном с векторами пространстве в нотации внешнего пространства выбранных двух векторов.

С операцией построения присоединенного к двум векторам трехмерного векторного ПСК-пространства связаны стандартные векторные операции и ряд специальных ПСК-операторов РВА, показанных в таблице 6 (здесь prBX и prBY 
- проекции вектора В на оси ПСК $\mathrm{X}^{0}=\mathrm{A}^{0}$ и $\mathrm{Y}^{0}=[\mathrm{A} \times \mathrm{B}]^{0}, \operatorname{prCXY}-$ проекция некоторого вектора С на плоскость векторов А и В, “ортогоны”- см. ниже):

Таблица 6 Векторные операции в пространстве ПСК двух векторов

\begin{tabular}{|c|c|c|c|}
\hline Оператор & \multicolumn{3}{|c|}{ Описание; ответ } \\
\hline Присоединенная СК & \multicolumn{3}{|c|}{ SystKoord PSK(vector A, vector B, double* prBX, double* } \\
\hline Репер АBC (\| $\left.\mathbf{A}^{*}, \mathbf{B}^{*}, \mathbf{C}^{*} \|^{*}\right)$ & \multicolumn{3}{|c|}{ V6D|V9D Reap2|3(A,B)= $\|\operatorname{Mtr}(\mathbf{A}, \mathbf{B})\|$ или $\|\operatorname{Mtr}(\mathbf{A}, \mathbf{B}, \mathbf{C})\|$} \\
\hline Проекция вектора В на Үํ ПСК & vector BortA(vector $\mathbf{A}$, vector $\mathbf{B})$ & \multicolumn{2}{|c|}{$\mathbf{B}_{\perp \mathbf{A}}=\operatorname{pr} \mathbf{B}_{\mathbf{Y}}$} \\
\hline Проекция вектора В на $\mathbf{X}^{0}$ ПСК & vector BonA(vector $\mathbf{A}$, vector $\mathbf{B})$ & \multicolumn{2}{|c|}{$\mathbf{B}_{\mathbf{A}}=\operatorname{pr} \mathbf{B}_{\mathbf{x}}$} \\
\hline Проекция вектора С на $\mathrm{X}^{0} \mathrm{Y}^{0}$ & \multicolumn{2}{|c|}{ vector ConAB(vector $\mathbf{A}$, vector $\mathbf{B}$, vector $\mathbf{C}$ ) } & $C_{A B}=p r C_{X Y}$ \\
\hline Проекция вектора С на $\mathbf{Z}^{0}$ ПСК & \multicolumn{3}{|c|}{ vector CortAB(vector $\mathbf{A}$, vector $\mathbf{B}$, vector $\mathbf{C}$ ) $\quad \mathbf{C}_{\perp A}$} \\
\hline Ортогоны векторов А и В & \multicolumn{3}{|c|}{ double ORTG(vectorA, vectorB, ${ }^{*}$ vector } \\
\hline
\end{tabular}

При построении алгоритмов решения прямых и обратных задач позиционирования вся семантика выполняемых в ПСК преобразований хорошо видна в пространстве, связанном с заданными векторами, в то время как все компоненты получаемых векторов "невидимо" вычисляются в исходной системе координат с отображением результата в метрике ПСК (см. рис. 2 и 3).

\section{Ортогонализация и проецирование векторов репера}

Для анализа и синтеза положений в пространстве ПСК репера векторов $\mathbf{A}, \mathbf{B}, \mathbf{C}$ часто нужны функции проектирования и ортогонализации AortB $(\mathbf{A}, \mathbf{B})$, $\operatorname{AonB}(\mathbf{A}, \mathbf{B}) \operatorname{BortA}(\mathbf{A}, \mathbf{B})$, BonA $(\mathbf{A}, \mathbf{B}), \operatorname{CortAB}(\mathbf{A}, \mathbf{B}, \mathbf{C}), \operatorname{ConAB}(\mathbf{A}, \mathbf{B}, \mathbf{C})$ и объединяющий их оператор Reaper( $\mathbf{A}, \mathbf{B}, \mathbf{C})$.

В частности, если репер векторов градиентов А,В,C используется для анализа отображений векторных пространств $\{\mathrm{x}, \mathrm{y}, \mathrm{z}\}$ и $\{\xi, \eta, \zeta\}$, то особую роль (см. [2] и рис. 5) играет вектор $\mathbf{C}_{\perp \mathbf{A B}}=\operatorname{CortAB}(\mathbf{A , B}, \mathbf{C})$. Он представляет собой вектор условного градиента для компоненты вектора $\zeta$, отображаемого градиентом $\mathbf{C}$, при условии отсутствия изменения компонентов $\xi, \eta$.

Эти операторы после построения ПСК для векторов его параметров $\mathbf{A , B}$ выполняют действия определения проекций и ортогональных векторов $\mathbf{A}_{\perp \mathbf{B}}, \mathbf{A}_{\mathbf{B}}$, $\mathbf{B}_{\mathbf{A}}, \mathbf{B}_{\text {Yпск }}, \mathbf{C}_{\perp \mathbf{A B}}$ и $\mathbf{C}_{\mathbf{A B}}$, связанных с векторами $\mathbf{A}, \mathbf{B}$ и $\mathbf{A} \times \mathbf{B}$ и направлениями осей ПСК (см. рис. 2, 3 и Табл. 6): ${ }^{2}$

$$
\mathbf{X}_{\text {ПСК }}^{0}=\mathbf{A}^{0}, \mathbf{Y}_{\text {ПСК }}^{0}=[\mathbf{A} \times \mathbf{B} \times \mathbf{A}]^{0} \text { и } \mathbf{Z}_{\text {ПСК }}^{0}=[\mathbf{A} \times \mathbf{B}]^{0} \text {; }
$$

Тогда

$$
\mathbf{B}_{\mathrm{A}}=\mathbf{B}_{\mathbf{Х п с к}}=\left(\mathbf{B} \bullet \mathbf{X}_{\text {ПСК }}^{0}\right) \cdot \mathbf{X}_{\text {ПСК }}^{0} \quad \text { и } \quad \mathbf{B}_{\perp A}=\mathbf{B}_{\text {Үпск }}=\left(\mathbf{B} \bullet \mathbf{Y}_{\text {ПСК }}^{0}\right) \cdot \mathbf{Y}_{\text {ПСК }}^{0} ;
$$

и

$$
\mathbf{A}_{\mathbf{B}}=\left(\mathbf{A} \bullet \mathbf{B}^{0}\right) \cdot \mathbf{B}^{0} \quad \text { и } \quad \mathbf{A}_{\perp \mathbf{B}}=\left(\mathbf{A} \bullet\left[\mathbf{B}^{0} \times \mathbf{Z}_{\text {ПСК }}^{0}\right]\right) \cdot\left[\mathbf{B}^{0} \times \mathbf{Z}_{\text {ПСК }}^{0}\right] .
$$

\footnotetext{
2 Поскольку обозначение (A,B) ниже использовано для обозначения плоскости, натянутой на векторы, то для скалярного и векторного произведений используются обозначения $(\mathbf{A \bullet B ) ~ и ~}[\mathbf{A} \times \mathbf{B}]$ соответственно. Операция нормирования вектора обозначается верхним индексом $\left({ }^{0}\right)$ у его имени.
} 
Проекции вектора $\mathbf{C}$ на ось $\mathbf{Z}_{\text {пск }}^{0}$ и на плоскость $\mathbf{A B}$ (рис.2) находятся просто скалярным произведением $\mathbf{C}_{\perp \mathbf{A B}}=\left(\mathbf{C} \bullet \mathbf{Z}_{\text {псК }}^{0}\right)$ и разностью $\mathbf{C}_{\mathbf{A B}}=\mathbf{C}-\mathbf{C}_{\perp \mathbf{A B}}$.

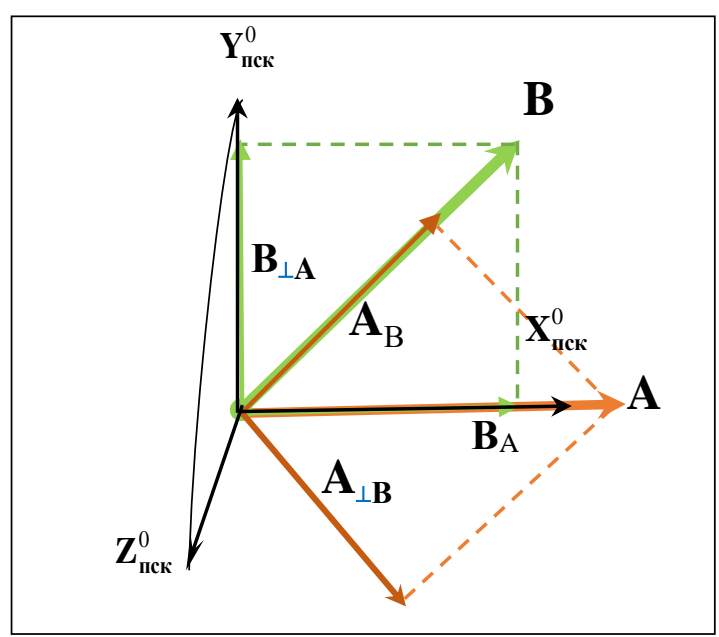

Рис. 3. Присоединенная система координат и “ортогоны” пары векторов А и В

Заметим, что пара специальных векторов (“ортогонов”) $\mathbf{A}_{\perp \mathbf{B}}$ и $\mathbf{B}_{\perp \mathbf{A}}$, присоединяемая к паре векторов $\mathbf{A}$ и $\mathbf{B}$, имеет важное линейно-алгебраическое значение: эти два вектора определяют геометрию строк обратной матрицы для двумерной матрицы векторов-строк системы линейных уравнений типа $\left\|\mathbf{A}^{\mathrm{T}} \mathbf{B}^{\mathrm{T}}\right\|^{\mathrm{T}} \cdot \mathbf{X}$ $=\mathbf{Y}$ (в обозначениях табл. 6 такая матрица векторов-строк градиентов величин Y выражается оператором репера градиентов $\operatorname{Reap}(\mathbf{A}, \mathbf{B})$ как $\|\operatorname{Mtr}(\mathbf{A}, \mathbf{B})\|)$.

Решения двумерной системы уравнений простым способом выражается и определяется через нормированную сумму векторов-ортогонов (см. рис. 3):

$$
\|\operatorname{Mtr}(\mathbf{A}, \mathbf{B})\| \bullet \mathbf{X}=\mathbf{Y} \Rightarrow \mathbf{X}=\left(\mathbf{A}_{\perp \mathbf{B}} /\left(\mathbf{A}_{\perp \mathbf{B}}\right)^{2}+\mathbf{B}_{\perp \mathbf{A}} /\left(\mathbf{B}_{\perp \mathbf{A}}\right)^{2}\right) \bullet \mathbf{Y} \text {. }
$$

Важность упомянутых операций ортогонализации в системе РВА связана и с тем обстоятельством, что при решении обратных задач геометрии они дополняют систему стандартных операций векторной алгебры нужными операциями семантического и метрического описания геометрии тех векторов твердого тела, которые не ортогональны выбираемым осям вращения.

При вращениях таких векторов вокруг неортогональных осей эти векторы движутся не по плоскости, а по поверхности некоторого конуса. По этой причине возникает необходимость присутствия в операторах PBA специальных операторов конических преобразований для выполнения вращений любых, а не только ортогональных векторов.

Заметим, что именно эта причина и породила моду использования кватернионов. Так же, как и у кватернионов, основная идея в системе операторов РВА - позволить формировать нужные действия на понятийном уровне описаний нужных геометрических преобразований и спрятать от пользователя их реализацию на векторно-метрическом уровне служебных программ. 
Однако система векторных операторов имеет гораздо больше возможностей для выполнения движений и преобразований геометрических объектов.

\section{ВЕКТОРНЫЕ АЛГОРИТМЫ КОНИЧЕСКИХ ПОВОРОТОВ}

Необходимость использования операторов косоугольных поворотов взаимно неортогональных векторов, как правило, вызвана техническими трудностями изготовления точно ортогональных направлений осей и элементов конструкции машины или датчиков конфигурационных углов ее кинематической схемы (например, при их креплении в труднодостижимых или взаимно удалённых местах конструкции). Для этого в системе РВА предусмотрен ряд операций для построения нужного положения вектора на конусе, намного превышающих возможности кватернионов по широте задач (Таблица 7):

\begin{tabular}{|l|c|}
\multicolumn{1}{c}{ Таблица 7} & Векторные операции на конусе \\
\hline \hline \multicolumn{1}{|c|}{ Оператор } & Описание; ответ \\
\hline \hline Поворот вектора вокруг вектора & $\mathrm{V}=\mathrm{PVV}(\mathbf{A}, \mathbf{B}, \mathbf{q}, \varphi)$ \\
\hline Отображение вектора на конусе с углом & $\mathrm{V}=\mathrm{VHAK}(\mathbf{B}, \mathrm{Fi}, \mathbf{C})$. \\
\hline Поворот вектора на конусе под другой вектор & $\mathrm{V}(\alpha \beta \gamma)=\mathrm{PVK}\left(\mathbf{A}, \mathbf{B}, \mathbf{C}, \mathrm{N}, \mathrm{Alf}, \mathbf{B}_{1}, \mathbf{B}_{2}, \mathbf{B}_{3}\right)$ \\
\hline Поворот вектора до совмещения с плоскостью & $\mathrm{D} 6 \mathrm{~V}=\mathrm{PVUPL}\left(\mathbf{A}, \mathbf{B}, \mathbf{C}, \mathbf{X}_{1}, \mathbf{X}_{2}\right)$. \\
\hline Поворот вектора до угла к другому вектору & $\mathrm{H}_{1}=\mathrm{VKU}\left(\mathbf{A}, \mathbf{B}, \mathbf{C},, \mathbf{U}, \mathrm{N}_{1}, \mathrm{H}_{2}\right)$ \\
\hline Определение оси и угла эйлерового поворота & $\mathrm{H}=\mathrm{EU}\left(\mathbf{A}_{1}, \mathbf{B}_{1}, \mathbf{A}_{2}, \mathbf{B}_{2}\right)$ \\
\hline
\end{tabular}

\section{Базовый оператор поворота вектора вокруг вектора}

Семантически наиболее важным оператором, лежащим в основе многих операций PBA, является оператор "PVV", выполняющий преобразование конического однопараметрического поворота заданного вектора вокруг другого заданного вектора на заданный угол. Операция такого преобразования гомоморфна популярной операции преобразования нормированного кватерниона $[4,5,19-25]$, которая геометрически реализует именно такой поворот в гиперкомплексной нотации векторных переменных. В данном случае эта операция удобно описывается и реализуется в привычных переменных обычного 3Dвекторного пространства и в переменных \{Вектор, Ось, Угол поворота\}.

Оператор конического «поворота вектора вокруг вектора» $\operatorname{PVV}(\mathbf{A}, \mathbf{B}, \varphi)$ обращается к оператору ортогонального осевого поворота путём построения и вращения на угол $\varphi$ вокруг оси $\mathbf{A}$ проекции поворачиваемого вектора $\mathbf{B}$ на плоскость $(\mathbf{X}, \mathbf{Y})$ ПСК - присоединенной к паре векторов, участвующих в описываемом повороте.

Орты осей $\mathbf{X}, \mathbf{Y}$, и $\mathbf{Z}$ присоединенной системы координат и компоненты вектора В в её осях показаны на рис. 4. Они соответственно равны:

$$
\mathbf{X}^{\mathbf{0}}=\mathbf{A}^{0}, \quad \mathbf{Y}^{0}=[\mathbf{A} \times \mathbf{B}]^{0} \times \mathbf{A}^{0}, \quad \mathbf{Z}^{0}=[\mathbf{A} \times \mathbf{B}]^{0}
$$

И

$$
\mathrm{B}_{\mathrm{X}}=\left(\mathbf{B} \bullet \mathbf{X}^{0}\right), \mathrm{B}_{\mathrm{Y}}=\left(\mathbf{B} \bullet \mathbf{Y}^{0}\right), \mathrm{B}_{\mathrm{Z}}=0 \text {. }
$$




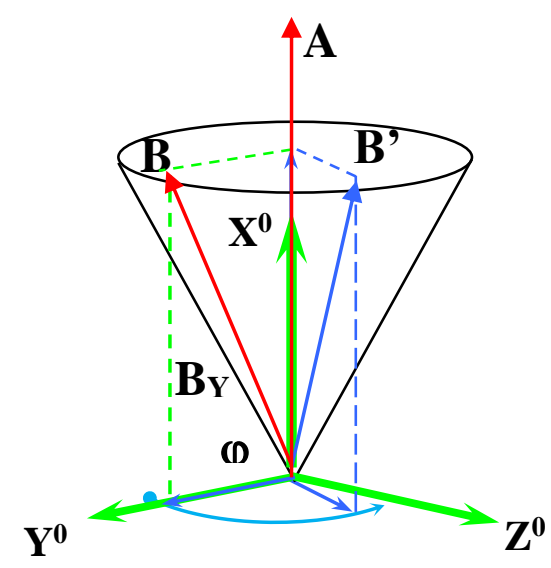

Рис. 4. Поворот вектора $\mathbf{B}$ вокруг вектора $\mathbf{A}$ на угол $\varphi$, описываемый векторным оператором PVV(A, B, $\varphi$ ) в системе координат, присоединенной к векторам $\mathbf{A}$ и $\mathbf{B}$.

Известно, что постановку и решение подобной задачи первым предложил Л.Эйлер в связи с доказанной им теоремой о существовании единственного («эйлерового») поворота твердого тела для реализачии любой нужной новой его ориентации в пространстве. В отличие от упомянутой теоремы предложенное Эйлером решение задачи осевого поворота осталось незамеченным. Решение этой задачи прославило О.Родриго, который много позже (в 1840 г.) предложил знаменитую «формулу поворота Родриго», в ее современной векторной записи и в параметрах рисунка имеющей вид (см., например, [23]):

$$
\mathbf{B}^{\prime}=\left(\mathbf{B} \cdot \mathbf{X}^{0}\right) \cdot \mathbf{X}^{0}+\left[\mathbf{X}^{0} \times \mathbf{B}\right] \cdot \sin \varphi+\left[\left[\mathbf{X}^{0} \times \mathbf{B}\right] \times \mathbf{X}^{0}\right] \cdot \cos \varphi .
$$

В наше время после публикации известной работы [4] для реализации операщчи осевого поворота в космической технике стала широко использоваться техника кватернионов. Алгебра кватернионов в кинематике твердого тела подробно описана и в упомянутой работе [23], где в переменных Родриго-Гамильтона показан эффективный метод формирования нужного кватерниона по заданным положениям векторов до $и$ после их требуемого поворота в пространстве базовой системы координат.

Вместе с тем семантически значительно более простое векторное представление операции осевого поворота, развитое в системе РВА в работах $[3,11,14]$, реализуется оператором «Поворот вектора вокруг вектора на угол ф» $\mathrm{PVV}(\mathbf{A}, \mathbf{B}, \varphi)$

В этом операторе последовательно выполняются операции: ортогонализация BortA (A,B), проектирующая исходный вектор $\mathbf{B}$ на ось $\mathrm{Y}^{0}$ ПСК, затем - поворот полученной проекции $\mathbf{B}_{\mathbf{Y}}$ на заданный угол $\varphi$ в плоскости $\left(\mathrm{Y}^{0}, \mathrm{Z}^{0}\right)$ ПСК и, наконеи, векторное сложение полученного вектора $\mathbf{B}_{Y}$ с вектором $\mathbf{B}_{\mathbf{X}}$ проекции исходного вектора $\mathbf{B}$ на направление оси вращения А.

Легко видеть, что в такой интерпретащии действий оператора $\mathrm{PVV}(\mathbf{A}, \mathbf{B}, \varphi)$ описывается выполнение геометрического содержания членов формуль Родриго.

Заметим, что представление осевых поворотов операторами PVV не менее лаконично и полно, а семантически оно более очевидно, чем их представление кватернионами. При этом линейным выражениям алгебры кватернионов соответствуют в программной реализачии РВА более простые и более богатые по структурным возможностям выражения алгебры векторных подстановок в формальные параметры операторов РВА, например, подстановки такого типа:

$$
\mathbf{R}=\operatorname{PVV}\left(\operatorname{PVV}\left(\left(\mathbf{A}+\left(\mathbf{B} \cdot \mathbf{C}^{0}\right) \cdot \mathbf{C}^{0}\right), \mathbf{D}, \varphi_{1}\right), \operatorname{PVV}\left(\mathbf{E},[\mathbf{F} \times \mathbf{C}]^{0} \cdot|\mathbf{G}|, \varphi_{2}\right), \varphi_{3}\right) .
$$

Иными словами, кватернионы и PVV отличаются лишь нотацией, но не содержанием. Однако в векторной нотации можно решить гораздо больше задач, чем в кватернионах. 
Эти вычисления в операторе $\operatorname{PVV}(\mathbf{A}, \mathbf{B}, \varphi)$ легко реализуются обращениями к операторам векторного и скалярного произведений векторов $\operatorname{Vvect} V(\cdot, \cdot)$ и $\operatorname{VscalV}(\cdot, \cdot)$ с соответствующим выбором нужных векторов построенной ПСК. Тогда после поворота на угол $\varphi$ новый вектор $\mathbf{B}^{\prime}$ в исходной системе координат равен векторной сумме: $\quad \mathbf{B}^{\prime}=\mathrm{B}_{\mathrm{Y}} \cdot \operatorname{Cos} \varphi \cdot \mathbf{Y}^{0}+\mathrm{B}_{\mathrm{Y}} \cdot \operatorname{Sin} \varphi \cdot \mathbf{Z}^{0}+\mathrm{B}_{\mathrm{X}} \cdot \mathbf{X}^{0}, \quad$ что записывается в операторном виде как $\quad \mathbf{B}^{\prime}=\operatorname{PVV}(\mathbf{A}, \mathbf{B}, 999, \varphi)^{3}$.

При обращении к PVV параметр q = 999 говорит о том, что задан именно угол $\varphi$. Другие варианты значения q предусматривают использование вместо угла его тригонометрических функций. Это удобно, когда нужный угол поворота определяется из результата скалярного и/или векторного произведения известных единичных векторов (без использования обратных тригонометрических функций) с определением представления получаемой четверти угла. Есть 3 способа:

$$
\mathbf{B}^{\prime}=\operatorname{PVV}(\mathbf{A}, \mathbf{B}, \cos (\varphi), \sin (\varphi)), \mathbf{B}^{\prime}=\operatorname{PVV}(\mathbf{A}, \mathbf{B}, \cos (\varphi), \pm 1), \mathbf{B}^{\prime}=\operatorname{PVV}(\mathbf{A}, \mathbf{B}, \pm 1, \sin (\varphi)) \text {. }
$$

\section{Операторы построения нужных конических движений}

Операторы типа PVV необходимы при решении прямых задач геометрии и кинематики. Геометрия операции, обратной заданному повороту вектора вокруг известной оси поворота, казалось бы, заключается в эйлеровой постановке задачи определения вектора оси и угла поворота, переводящих вектор одной ориентации в этот же или иной вектор другой ориентации и позиции. Однако у пары векторов в такой задаче не имеется единственного решения - существует множество возможных положений осей любых нужных конусов в пространстве.

Напомним, что знаменитая теорема Эйлера сформулирована для твердого тела и для постановки обратной задачи нужны по крайней мере две пары одинаковых векторов в разных их позициях и ориентациях. Иными словами, в постановке обратной задачи для единственного вектора нужно дополнительно задать либо конус поворота, либо угол поворота, либо дополнительный параметр вектор результата поворота. Для решения обратных задач в системе PBA имеются операторы и функции определения векторов и углов на конусе (их аналоги для поиска углов поворотов и векторов не на конусе, а на плоскости получаются, как частный случай, выбором ортогональных векторов А и В). Наиболее частным случаем обратной задачи свободного вектора является задача поиска угла поворота вектора вокруг заданной оси до определенной плоскости, содержащей ось поворота, или - до получения заданного угла между поворачиваемым вектором и некоторым определенным вектором.

\footnotetext{
3 Этот оператор простым поворотом в ПСК реализует векторно-геометрическое содержание формулы Гамильтона-Родрига - вне использования техники кватернионов.
} 
Отображение вектора на конус

Функция $\mathbf{C}_{\mathrm{A}}=\mathrm{VtoK}(\mathrm{A}, \mathrm{Fi}, \mathbf{C})$ возвращает отображение $\mathbf{C}_{\mathrm{A}}$ вектора $\mathbf{C}$ на конус, заданный вектором оси А и углом раствора Fi (рис. 5).

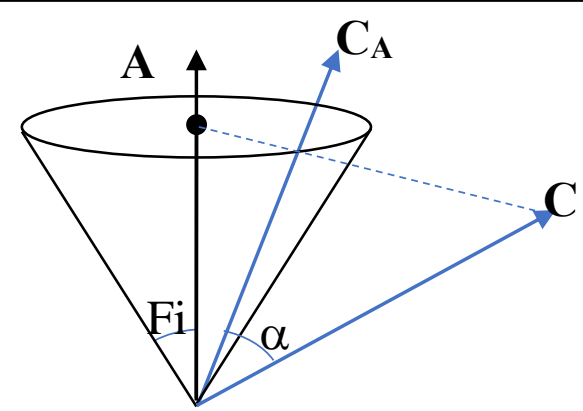

Рис. 5. Отображение вектора на конус

Такое отображение нельзя называть проекцией, поскольку вектор $\mathbf{C}_{\mathbf{A}}$ не изменяет размера вектора С. Геометрически оно соответствует повороту вектоpa C к конусу (A.Fi). Однако в действительности эта операция выполняется поворотом единичного вектора оси $\mathbf{A}^{\mathbf{0}}$ до поверхности конуса в сторону заданного вектора $\mathbf{C}$ с последующим растяжением полученного вектора до размеров этого вектора С. Подобная операция удобна для построения нужного конуса, заданного вектором его оси и углом раствора с построением образующего конус вектора нужной длины. Это описывается просто набором вложенных операторов:

$$
\mathbf{C}_{\mathrm{A}}=\operatorname{VtoK}(\mathbf{A}, \mathbf{F i}, \mathbf{C})=\operatorname{VuS}(\operatorname{PVV}(\operatorname{VEd}(\mathbf{A}), \operatorname{VvectV}(\mathbf{A}, \mathbf{C}), 999, \text { Fi }), \operatorname{VMod}(\mathbf{C})) \text {; }
$$
Приведенная формула демонстрирует стиль работы с данными операторов PBA.

\section{Угол поворота вектора под вектор}

Эта функция обращением к оператору $\varphi=\operatorname{UKpodV}(\mathbf{A}, \mathbf{B}, \mathbf{C})$ вычисляет угол поворота вектора $\mathbf{B}$ до ближайшего к $\mathbf{C}$ вектора $\mathbf{B}_{1}$ в плоскости векторов $\mathbf{A}, \mathbf{B}$ и возвращает получаемый угол $\varphi$, как показано на рис. 6.

Заметим, что вектор $\mathbf{C}$ не должен совпадать с вектором $\mathbf{A}$, иначе $\varphi=0$. Имеем: $\quad \mathbf{B}_{1}=\operatorname{PVV}(\mathbf{A}, \operatorname{VvectV}(\mathbf{A}, \mathbf{C}), \operatorname{VskalV}(\operatorname{VMod}(\mathbf{A}), \operatorname{VMod}(\mathbf{B})), 1)$. Здесь: $\quad$ вектор ${ }^{\wedge} \wedge$ ось поворота $\wedge^{\wedge} \cos \left(\right.$ угла поворота) $\wedge_{\text {знак } \sin =+ \text {. }}$

$$
\varphi=\operatorname{VugV}\left(\operatorname{BortA}(\mathbf{A}, \mathbf{B}), \operatorname{BortA}\left(\mathbf{A}, \mathbf{B}_{1}\right), \mathbf{A}, 1\right),
$$

VugV - функция вычисления угла между векторами и его четверти (см. ниже).

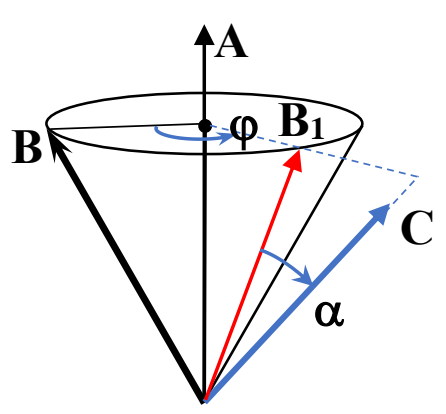

Рис. 6. Построение вектора на конусе 


\section{Поворот вектора до совмещения с плоскостью}

Легко видеть (см. рис. 6), что задача определения угла ф поворота вектора $\mathbf{B}$ вокруг вектора $\mathbf{A}$ для совмещения вектора $\mathbf{B}$ с плоскостью векторов $(\mathbf{A , C )}$ совпадает с задачей поворота вектора под вектор и решается обращением к предыдущей функции. Отдельная функция $\mathbf{B}_{1}=\mathrm{VKtoPL}(\mathbf{A}, \mathbf{B}, \mathbf{C})$ удобна, когда нужно определить вектор результата совмещения с плоскостью.

\section{Построение векторов на конусе под углом к другому вектору}

Важный для приложений оператор «Векторы - Конус - Угол» (рис. 7):

$$
\left\{\psi_{1}, \psi_{2}, \psi_{0}\right\}=\operatorname{VKU}\left(\mathbf{A}, \mathbf{B}, \mathbf{C}, \mathrm{N}, \alpha,{ }^{*} \mathbf{B}_{1},{ }^{*} \mathbf{B}_{2}\right)^{4}
$$

- Построение векторов и углов поворота на конусе - определяет на конусе с его осью $\mathbf{A}$ и углом образующего вектора $\mathbf{B}$ два таких вектора $\mathbf{B}_{1}, \mathbf{B}_{2}$, что они составляют требуемый угол $\alpha$ (при $\mathrm{N}=999$,) с заданным вектором С. Если задан не сам угол $\alpha$, a $\cos \alpha$ (что бывает удобно при определении нужного угла с помощью скалярного произведения известных векторов), то $\mathrm{N}=\cos \alpha$, а знак параметра $\alpha$ определяет знак угла $\alpha$ (в предположении, что $\alpha \in 0 \ldots \pm \pi)$. Оператор возвращает вектор 3 углов $\psi_{1,2}=\mathrm{BA}_{0} \mathrm{~B}_{1,2}$ и $\psi_{0}=\mathrm{BA}_{0} \mathrm{C}_{0}$.

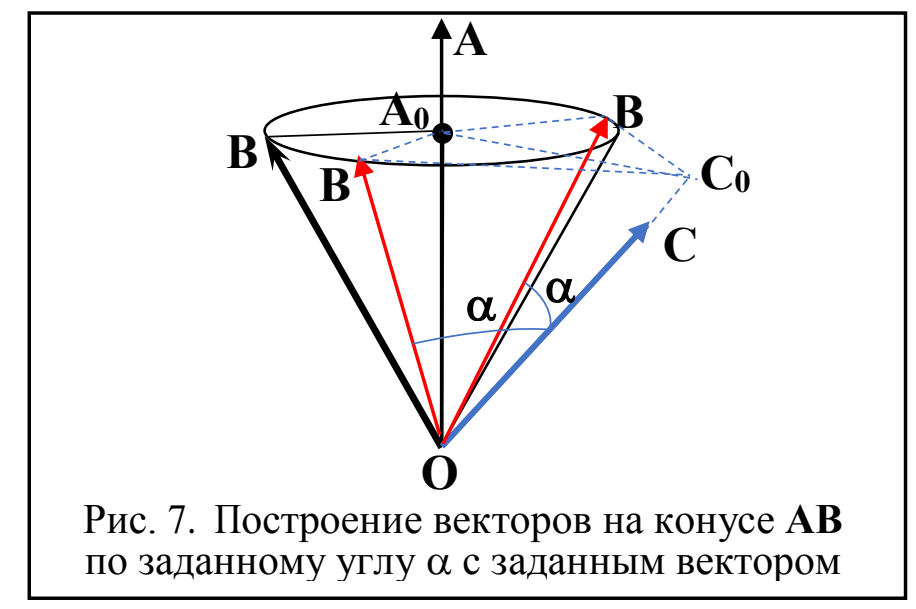

Эта полезная операция определяет векторы пересечения двух конусов. Она чаще всего необходима для определения углов построения требуемой ориентации вектора в пространстве способом выполнения фиксированной последовательности «трех эйлеровых поворотов» вокруг связанных с вектором осей типа поворотов на небесно-механические углы Эйлера - прецессии, нутации и собственного вращения, или - на корабельно-самолетные углы Крылова - курс, дифферент (тангаж), крен, или на ракетные углы - тангаж, курс, крен.

В системе PBA с помощью этого оператора реализованы стандартные операторы «двухпараметрического разворота» и «трехпараметрического разворота» векторов твердого тела вокруг заданных связанных с телом осей для

\footnotetext{
4 Здесь и везде символ «*» в описании формального параметра оператора для удобства читателя показывает на вычисляемый оператором параметр (одновременно с этим в такой записи в языке С подразумевается возвращение найденного параметра по указанному при обращении к оператору адресу).
} 
направления нужным образом во внешнем пространстве связанной с телом приборной оси (двухпараметрический разворот) или осей связанной системы координат прибора или тела (трехпараметрический разворот).

Операция VKU c ее семантикой геометрии пересечения двух конусов имеет два ответа, что аналитически выражается решением некоторого квадратного уравнения. Но искомые векторы и углы поворота могут быть найдены просто геометрически. Для этого надо, определив углы между заданными векторами A, В и С, операторами проекции $\operatorname{BonA}(\mathbf{A}, \mathbf{B})$ и ортогона $\operatorname{BortA}(\mathbf{A}, \mathbf{B})$ определить вектор оси конуса $\mathbf{O A}_{1}$ вместе с размером радиуса этого основания $\mathbf{A}_{0} \mathbf{B}$ (см. рис. 6). Затем оператором $\operatorname{BortA}(\mathbf{A}, \mathbf{C})$ определить вектор $\mathbf{O C}_{0}$ в плоскости основания конуса на уровне образующего его вектора $\mathbf{B}$ вместе с углом $\psi_{0}=\angle \mathrm{BA}_{0} \mathrm{C}_{0}$. Тогда из треугольника $\mathrm{OB}_{1} \mathrm{C}_{0}$ с двумя известными сторонами и углом между ними определяется длина вектора $\mathbf{B}_{\mathbf{1}} \mathbf{C}_{1}$, что, в свою очередь, позволяет из угла $\angle \mathrm{B}_{1} \mathrm{~A}_{0} \mathrm{C}_{0}$ треугольника $\mathrm{B}_{1} \mathrm{~A}_{0} \mathrm{C}_{0}$ определить искомые углы $\psi_{1}=\angle \mathrm{BA}_{0} \mathrm{~B}_{1}$ и $\psi_{2}=\angle \mathrm{BA}_{0} \mathrm{~B}_{2}$.

Знание этих параметров дает возможность применением оператора PVV (поворот вектора вокруг вектора) определить искомые векторы $\mathrm{B}_{1}=\mathrm{PVV}\left(\mathrm{B}, \mathrm{A}, \psi_{1}\right)$ и $\mathrm{B}_{2}=\operatorname{PVV}\left(\mathrm{B}, \mathrm{A}, \psi_{2}\right)$.

\section{Определение оси и угла эйлерового поворота пары векторов}

Поскольку положение и ориентация твердого тела в пространстве определяется положением и ориентацией трех точек тела или любой пары его векторов, то для определения параметров конечного эйлерового поворота тела не только в случае присутствия неподвижной точки, но и для более общего случая винтового движения тела или самого общего случая суммирования вращения с поступательным движением выбранной точки тела - во всех этих случаях необходимо иметь два пространственных положения одной и той же пары взаимно связанных вектора.

Однако всегда два несоприкасающихся позиционных Poz-вектора можно заменить на два вектора с их общим началом в выбранной точке («полюса») тела и с вычисляемой позицией этой точки во внешней системе координат. После этого задача построения параметров конечного поворота и позиционного сдвига тела для совмещения исходной и целевой его позиций заключается в определении Poz-вектора позиции и направления эйлеровой оси в связанной и/или внешней системах координат вместе с определением угла требуемого конечного поворота при предположении поступательного перемещения выбранного полюса тела для совмещения начал обеих пар векторов в одной конечной точке. Геометрия векторного решения задачи построения оси и угла конечного эйлерового 
поворота двух пар векторов с общим началом в целевой точке движения определяется следующими (см. рис. 8) соображениями.

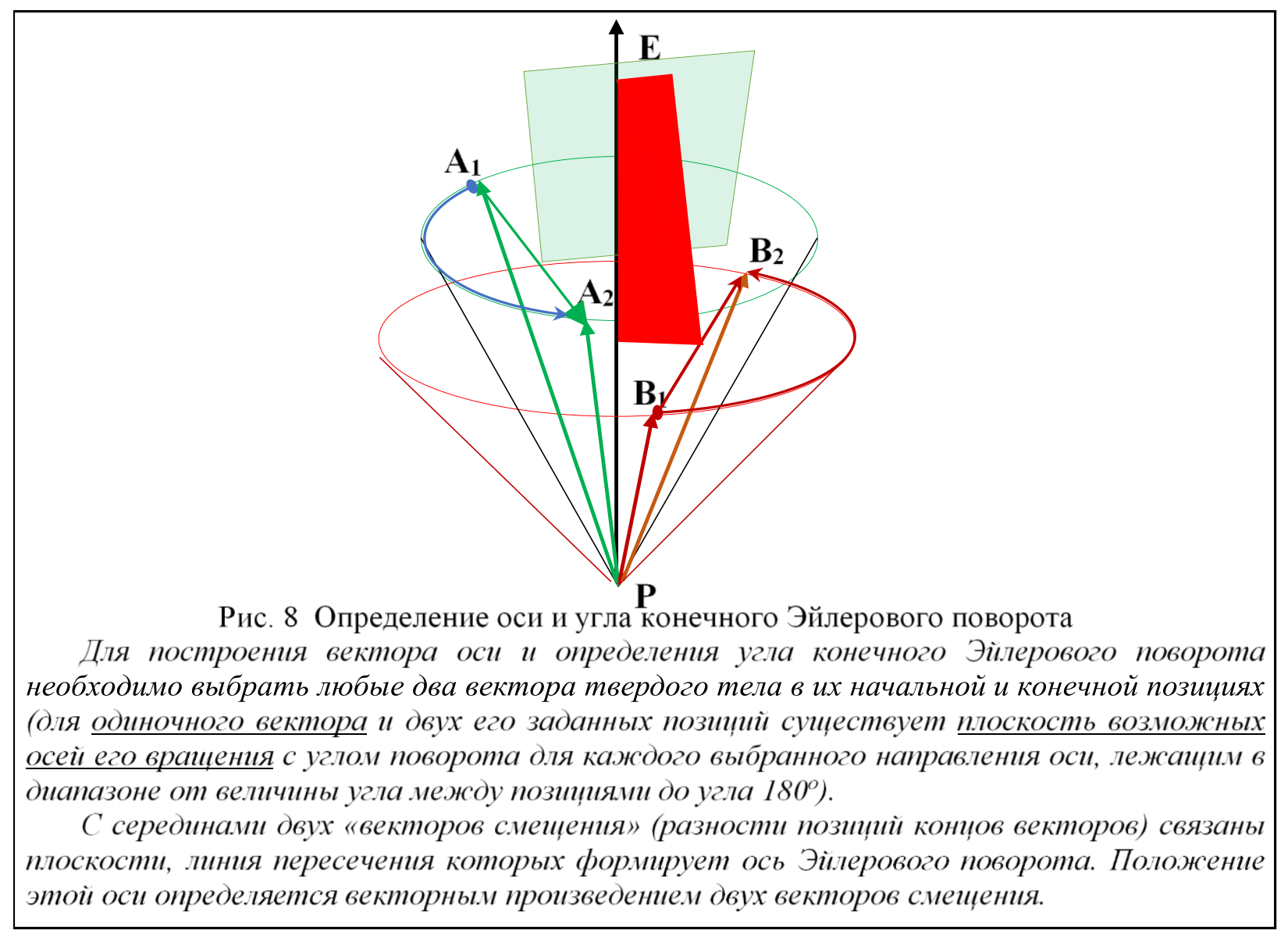

Начальная и конечная позиции любой пары векторов твердого тела при его конечном повороте принадлежат двум соосным конусам с их общей Эйлеровой осью. Важную роль в решении обратной задачи Эйлерового поворота поиска величины угла и расположения его оси - имеют два вектора (назовем их «векторами смещения») разностей векторов начальной и конечной позиции для пары выбранных векторов твердого тела. Они представляют собой хорды дуг следов эйлерового вращения концов каждого из векторов на этих конусах. Очевидно, что эти хорды и дуги принадлежат параллельным плоскостям с их нормалями, коллинеарными вектору эйлеровой оси конусов.

Положение эйлеровой оси в пространстве в этом общем случае определяется из следующего утверждения относительно упомянутых векторов хорд дуг эйлерового поворота (вытекающего из необходимости равных радиусов от оси поворота до границ каждой хорды):

Эйлерова ось представляет собой линию пересечения двух плоскостей, ортогональных векторам смещения и проходящих через их середины.

Такие плоскости содержат все возможные оси поворотов каждого из векторов между двумя заданными его позициями. Их пересечение удовлетворяет условию одинаковости искомого перемещения векторов твердого тела. И по- 
скольку векторы смещения являются нормалями к плоскостям возможных осей поворота каждого из векторов, то направление искомой оси конечного эйлерового поворота определяется векторным произведением векторов смещения. Угол эйлерового поворота можно искать из решения радиальных треугольников, но легче его найти описанным выше оператором VKU из геометрического условия постоянства углов между векторами твердого тела.

Таким образом, для выбранной пары $\mathbf{A}$ и $\mathbf{B}$ векторов с известным углом $\gamma$ между ними и заданными позициями $\mathbf{A}_{1}, \mathbf{B}_{1}$ начального и $\mathbf{A}_{2}, \mathbf{B}_{2}$ конечного их положения в пространстве алгоритм определения параметров оси $\mathbf{E}$ и угла $\psi$ результирующего конечного поворота (вне зависимости от возможных способов позиционного перемещения тела между этими позициями) описывается следующими операторами PBA:

$$
\begin{gathered}
\mathrm{E}=\operatorname{VvectV}\left(\operatorname{VmV}\left(\mathbf{A}_{2}, \mathbf{A}_{\mathbf{1}}\right), \operatorname{VmV}\left(\mathbf{B}_{2}, \mathbf{B}_{1}\right)\right) ; \\
\left\{\psi_{1}, \psi_{2}\right\}=\operatorname{VKU}\left(\mathbf{E}, \mathbf{A}_{\mathbf{1}}, \mathbf{B}_{2}, \operatorname{VscalV}\left(\mathbf{A}_{\mathbf{1}}, \mathbf{B}_{1}\right), 1, * \mathbf{A}_{21}, * \mathbf{A}_{22}\right) .
\end{gathered}
$$

Из двух решений $\left(\mathbf{A}_{2 \mathrm{i}}, \psi_{\mathrm{i}}\right)$ выбирается удовлетворяющее условию $\mathbf{A}_{2 \mathrm{i}}=\mathbf{A}_{2}$. Эти соотношения реализуют оператор PBA: $\mathrm{H}=\{\mathbf{E}, \psi\}=\mathrm{EU}\left(\mathbf{A}_{\mathbf{1}}, \mathbf{B}_{1}, \mathbf{A}_{2}, \mathbf{B}_{2}\right)$.

Важно упомянуть отличие рассмотренной выше задачи конечного поворота от задачи построения гладкого движения тела с вращением между его заданными начальной и конечной позициями. В таком движении в силу известных теорем теоретической механики (см., например, [16]) однозначными параметрами являются лишь краевые позиции и найденные параметры оси и угла конечного поворота. Для учета времени и сил надо выбрать закон соотношений приращений угла du на пути dS на нужной траектории полюса $\mathrm{P}$.

\section{ОПЕРАТОРЫ ДВИЖЕНИЯ С ПОВОРОТОМ}

Bсе описанные выше операторы РВА формируют преобразование геометрии конечного состояния векторных направлений и позиций. Для перехода к задачам построения параметров и точек нужного движения между двумя заданными состояниями векторов ${ }^{5}$ при фиксированных параметрах конечного Эйлерового поворота в РВА принята концепция построения нужной геометрии траектории движения фиксированной точки векторного множества (его «полюса»), оставляя за скобками операторов РВА формирование закона движения вдоль принятой траектории.

В такой постановке задачи построения движения легко разделяются на хорошо изученные теоретической механикой задачи:

\footnotetext{
${ }^{5}$ B операторах PBA независимым параметром движения выбрана длина пути $\mathrm{S}$ фиксированной точки тела, а не время $\mathrm{t}$ момента движения. Это позволяет реализовать чисто геометрический подход к построению рисунка движения $\mathrm{S}(\mathrm{x}, \mathrm{y})$, оставляя скорость траекторного движения $\mathrm{V}(\mathrm{S})$ независимым внешним параметром для операторов РВА. Таким способом операторы РВА позволяют моделировать разную кинематику или динамику полюса движения вдоль построенной траектории.
} 
- задачи плоского движения (эйлерова ось конечного поворота является нормалью плоскости, содержащей всю траекторию пути полюса);

- задачи винтового движения (прямая траектория пути полюса коллинеарна эйлеровой оси требуемого конечного поворота);

- задачи общего вида пространственного движения с вращением (когда эйлерова ось конечного поворота на всех точках пути полюса образует некоторый угол с траекторией этого пути). Заметим, что в соответствии с теоремами Шаля и Моцци [25] все случаи такого движения специальным выбором переменного полюса в твердом теле сводятся к мгновенному винтовому движению с осью Эйлера, касательной к траектории выбранного пути полюса.

\section{Операторы плоских транспортных движений}

В задачах плоского движения с поворотом большой круг задач связан с построением движения транспортных средств по плоской (или мгновенноплоской) опорной поверхности. В связи с этим в системе операторов РВА с использованием формализма ПСК предусмотрены специальные операторы для построения фиксированных типов плоского поворотного движения.

\section{Оператор движения по кругу}

Для Poz-векторов направлений $\mathbf{A}$ и $\mathbf{B}$ в начальной и конечной позициях полюса робота (например, центра масс) из треугольника $\mathrm{ABE}$ (рис. 9) оператор

$$
\mathrm{H}=\mathrm{ARC}(\mathbf{A}, \mathbf{B}, \mathrm{R})
$$

определяет в Н-ответе позицию эйлеровой оси $\mathbf{E}$ и угол конечного поворота для движения полюса вдоль дуги окружности с вектором нужного радиуса R.

\section{Оператор рельсового поворота}

«Переходная дуга» переменной кривизны рельсовых путей формируется для гладкого безударного изменения смены направления движения на стыках прямых и кривых участков. В PBA для этой цели разработан оператор (рис. 9)

$$
\{\mathrm{X}, \mathrm{Y}, \psi\}=\operatorname{Kornu}(\mathrm{R}) \text {, }
$$

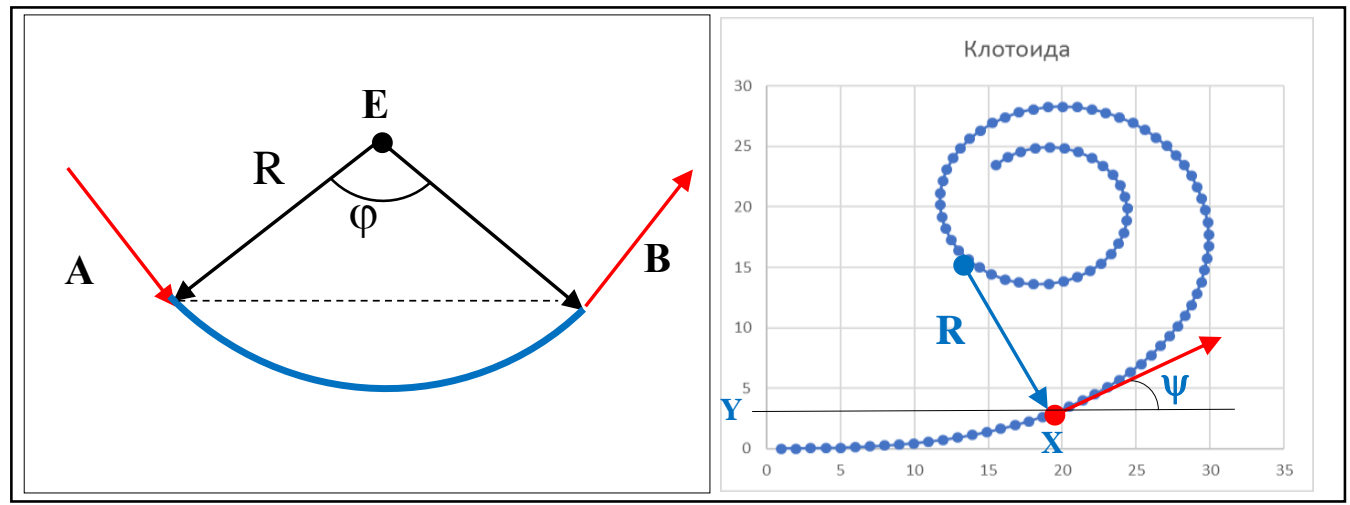

Рис. 9. Круговое $\mathrm{ARC}(\mathbf{A}, \mathbf{B}, \mathrm{R})$ движение и «радиодальная спираль» $\mathrm{Kornu}(\mathrm{R})$ 
который определяет точку конца переходной дуги в виде Клотоиды Корню («радиодальной спирали» с величиной кривизны, пропорциональной длине пути дуги [18]).

Клотоида в этом операторе строится от начала до точки заданного радиуса кривизны пути, измеряемого в единицах длины корпуса экипажа. Возвращается D3V-вектор: X,Y-координаты в этих же единицах и угол $\psi$ курса в конце дуги.

\section{Оператор формирования гладких траекторий}

$\mathrm{C}$ использованием операторов $\operatorname{Kornu}(\mathrm{R})$ и $\mathrm{ARC}(\mathbf{A}, \mathbf{B}, \mathrm{R})$ оператор PBA

$$
\operatorname{Tr}=\operatorname{EvenWay}(\mathbf{S}, \mathbf{F}, \mathbf{R})
$$

формирует фиксированной длины массив $\operatorname{Tr}$ точек гладкого пути между Pozвекторами старта $\mathbf{S}$ и финиша $\mathbf{F}$, вставляя круговое $(\mathbf{A}, \mathbf{B}, \mathbf{R})$ движение между участками Клотоид с радиусами $\mathrm{R}$ и суммой углов $\varphi+2 \psi$ в граничных точках.

\section{Оператор вальсирующего движения}

Вальсирующее движение для вектора А на заданной операторами Коти и $\mathrm{ARC}$ траектории $\operatorname{Tr}$ плоского или пространственного движения полюса формируется в каждой точке его поворотом на угол du вокруг вектора $\mathbf{E}$ на каждом шаге пути. Это выполняется следующим оператором PBA: $\operatorname{Vals}(\operatorname{Tr}, \mathbf{E}, \mathbf{A}, \mathrm{du})$.

\section{Оператор винтового движения}

Отличающим свойством винтового движения является коллинеарность векторов направления движения $\mathbf{S}$ и Эйлеровой оси $\mathbf{E}^{6}$. Параметр локального поворота $\varphi$ на пути $\mathrm{s}$ определяется или функцией $\varphi(\mathrm{s})$, или винтовым параметром «шаг винта» - длиной пути $S_{2 \pi}$ сопутствовавшего поворота на $2 \pi$. Для работы с винтовыми движениями развита технология бикватернионов [20-22, 24].

B PBA оператор VINT реализуется простым соединением PVV со сдвигом $\mathrm{s}(\varphi)$ :

$$
\operatorname{VINT}(\mathrm{E}, \mathrm{B}, \varphi, \mathrm{s})=\operatorname{PVV}(\mathrm{E},(\mathrm{B}=\mathrm{VpV}(\mathrm{B}, \mathrm{VuS}(\mathrm{VEd}(\mathrm{E}), \mathrm{s}))), 999, \varphi) \text {. }
$$

\section{ОПЕРАТОРЫ МЕТРИКИ УГЛОВ}

Хотя эти операторы прежде всего необходимы на спрятанном от пользователя уровне реализации исполнения операторов РВА, они, тем не менее, открыты и для использования при необходимости на уровне пользователя. Их устройство довольно очевидно, однако имеются и некоторые обстоятельства, учитываемые в РВА при реализации метрических операторов.

\footnotetext{
6 Заметим, что именно это обстоятельство и породило необходимость развития техники кватернионов (повороты направлений векторов) в технику дуальных кватернионов (повороты и сдвиги векторов) $[5,20,21,24]$. Описываемые операторы PBA и операторы векторных преобразований в пространстве гиперкомплексных чисел взаимно отображаемы друг в друга.

Выше было показано, что всё, выполнимое в технике кватернионов, выполнимо и в системе РВА. В частности, винты и более общие типы движения намного более просто реализуются в РВА стандартными векторными средствами.
} 
Геометрия и структура углов между позиционируемыми D6V-векторами и D9V-векторами заметно отличаются от стандартной геометрии линейных или сферических углов между свободными D3V-векторами. Это связано с необходимостью определения семантики операций с парой позиционных векторов и разработки специальных операторов для семантически удобного определения характерных углов между удаленными элементами геометрических объектов.

Другое, более стандартное обстоятельство связано с обычной необходимостью определения не только главного значения угла между двумя заданными направлениями, но и спецификой заранее не определенных соглашений о присвоении углу правильного знака с привязкой его значения к одному из четырех выбираемых типов угловых диапазонов:

Diap $\alpha= \pm\left(0^{\circ} . .360^{\circ}\right)$, Diap $\alpha= \pm\left(0^{\circ} . .180^{\circ}\right)$, Diap $\alpha=\left(0^{\circ} . .180^{\circ}\right)$ и Diap $\alpha= \pm\left(0^{\circ} . .90^{\circ}\right)$.

Очевидно, что при определении угла с помощью обратных тригонометрических функций знак и четверти угла определены однозначно знаками аргументов этих функций. Поэтому при таком построении алгоритма оператора вычисления угла можно не учитывать контекст и семантику отношений получаемого угла с соседними линиями или геометрическими объектами в рассматриваемом пространстве. Эта семантика важна лишь вне тригонометрического оператора.

Вместе с этим в РВА и простые функции, и более сложные описанные выше функции по необходимости привязаны к операционной семантике с контекстом пользователя (например, при формировании последним геометрического содержания операторов алгоритма решения задачи) и соглашениями с ним о способе выбора направления и четверти углов в пространстве. Причем очевидно, что эта связь и семантически, и алгоритмически различна для случаев простого вычисления углов между векторами и для случаев суммирования углов из разных источников в процессе их осевых векторных преобразований.

В первом случае в операторе РВА для определения угла между двумя векторами пользователю предоставляется возможность выбором третьего вектора указать сторону полупространства, содержащую выбранное положительное направление оси отсчета угла. Во втором случае в операторах сложения углов на множестве векторов с их известными положительными направлениями отсчета углов у пользователя должно быть средство реализации логики «укладывания» суммарного угла в желаемый диапазон результата.

С этими обстоятельствами в системе РВА для определения величины векторных углов, кроме упомянутых выше операторов конических угловых поворотов векторов, используются процедуры VugV(), UTrngl и UDiap() (см. таб. 7), в алгоритмах реализации которых предусмотрен синтаксис получения внешней информации об используемых в контексте программы положительных направлениях осей и выбранных типов диапазонов углов. 


\begin{tabular}{|c|c|}
\hline Операции вычисления вектс & углов \\
\hline Оператор & Описание; ответ \\
\hline Определение угла между векторами А и В с осью C & $\varphi=\operatorname{VugV}(A, B, C, N)$ \\
\hline Приведение угла в диапазон & $\varphi=\operatorname{UDiap}(U, N)$ \\
\hline Определение углов треугольника с известными сторонами & $\varphi=U \operatorname{Trng} \mid(a, b, c)$ \\
\hline Определение угла вектора с конусом А, $\varphi$ & $\alpha=\operatorname{UVtoK}(\mathbf{A}, \varphi, \mathbf{C})$ \\
\hline
\end{tabular}

Определение угла между векторами

Функция $\operatorname{Vug} \mathrm{V}(\mathbf{A}, \mathbf{B}, \mathbf{C}, \mathbf{N})$ "Определение угла между векторами А $и$ В с осью C" возвращает угол с его знаком в указанном при обращении диапазоне углов. Третий задаваемый вектор $\mathbf{C}$ нужен для выбора полупространства, содержащего положительное направление оси вращения вектора А в сторону к $\mathbf{B}$ (вектор $\mathbf{C}$ не должен принадлежать плоскости векторов $\mathbf{A , B ) . ~ Е с л и ~} \mathbf{C}$ - нулевой вектор VNul, то угол лежит в диапазоне $0 \ldots \pi$.

Признак N задает стандартный в РВА номер диапазона угла:

$\mathrm{N}=0$ : угол лежит в диапазоне $0 \ldots \pi$.

$\mathrm{N}=1$ : угол лежит в диапазоне $0 \ldots+\pi$ и $0 \ldots-(\pi-0.000001)$.

$\mathrm{N}>1$ : угол лежит в диапазоне $0 . . .(2 \pi-0.000001)$.

Подобный признак используется во многих операторах определения углов.

\section{Приведение угла в диапазон}

Эта операция необходима в случаях получения угла $U$ из суммы углов:

$$
\varphi=\operatorname{UDiap}(\mathrm{U}, \mathrm{N}) \text {, }
$$

где $\mathrm{N}$ - номер диапазона угла. Возвращается угол ф в указанном при обращении номере диапазона $\mathrm{N}$.

Операция "Углы треугольника"

Оператор $\operatorname{TreUgl}(\mathrm{ab}, \mathrm{bc}, \mathrm{ac})$ определяет по теореме косинусов углы треугольника abc с известными размерами длин его сторон (знаки сторон не учитываются). Возвращается D3V-вектор углов $\{\mathrm{abc}, \mathrm{bca}, \mathrm{cab}\}$ (все возвращаемые углы положительны). Если одна из сторон не существует (имеет значение 999 или 999.) или треугольник с такими сторонами невозможен, то возвращается несуществующий вектор VNot=\{999.,999.,999. $\}$.

\section{Определение угла вектора с конусом}

Для удобства определения угла между вектором $\mathbf{C}$ и конусом с осью $\mathbf{A}$ и углом $\varphi$ в РВА используется оператор-функция, аналог оператора VtoK:

$$
\alpha=\mathrm{UVtoK}(\mathbf{A}, \boldsymbol{\varphi}, \mathbf{C}) \text {. }
$$

Эта функция вычисляет и возвращает угол $\alpha$ от вектора $\mathbf{C}$ до ближайшего вектора на поверхности конуса (см. выше рис. 5), что реализуется обращением к описываемой выше функции $\mathbf{C}_{1}=\operatorname{VtoK}(\mathbf{A}, \mathrm{Fi}, \mathbf{C})$ и вычислением разности $\alpha_{\min }=\left(\operatorname{VugV}\left(\mathbf{A}, \mathbf{C}_{1}, \mathrm{VNul}, 1\right)-\mathrm{Fi}\right)$. 
Несложные рассуждения показывают, что в этой разности значения получаемых углов всегда реализуются в диапазоне $0 \pm \pi$, и обращение к функции $\operatorname{UDiap}\left(\alpha_{\min }, 1\right)$ поэтому здесь не требуется.

\section{ОПЕРАТОРЫ МЕТРИКИ РАССТОЯНИЙ}

Линейные расстояния между объектами векторного пространства всегда связаны с вычислением модуля вектора расстояния. Этот вектор можно сформировать либо как вектор разности двух известных Poz-векторов, либо в виде проекции известного вектора на нормаль к известному векторному объекту вектору, плоскости, конусу. Эти простые операции для свободных D3V-векторов с общим началом последних лишь немного усложняются для позиционируемых D6V-векторов с разнесенными в пространстве позициями их начал и заметно усложняются возникновением ситуации перекрещивания направлений векторных линий, отсутствующей в пространстве свободных векторов.

Перекрещивание направлений векторных линий (двухсторонних продолжений линии направления вектора) не всегда означает перекрещивания самих взаимно удаленных векторов. Поэтому возникает логика определения разных расстояний между векторами. Их можно выделить несколько типов:

- «позищионное расстояние» векторов: разность D3-векторов позиций их начал;

- «концевое расстояние» векторов: разность D3-векторов позиций их концов;

- «целевое расстояние» суммы Poz-векторов: сумма всех 4 векторов в составе двух Poz-векторов определяет D3-вектор цели этой суммы;

- «минимальное расстояние» векторов: разность векторов ближайших точек;

- «расстояние скрещивания» векторов: расстояние перекрещивания или пересечения векторных линий;

- «расстояние до скрещивания» с другим вектором: расстояние от позиции начала вектора вдоль его векторной линии (>0 - в сторону вектора) до точки его перекрещивания или пересечения с другим вектором.

Для реализации вычислений таких расстояний в системе РВА предусмотрены операторы, показанные в табл. 8.

Оператор определения расстояния между векторными D3V-точками

Этот простейший базовый оператор широко используется в содержании многих операторов РВА. Он заключается в определении модуля вектора разности D3V-векторов точек: $\quad \operatorname{dist}=\operatorname{VdoV}(\mathbf{A}, \mathbf{B}))=\operatorname{VMod}(\operatorname{VmV}(\mathbf{A}, \mathbf{B}))$.

Если векторы равны, возвращается нулевой вектор VNul. Если один из векторов не определен, то в качестве ответа возвращается неопределенный вектор.

\section{Оператор определения расстояния D3V-точки до плоскости PL}

Таких операторов в системе PBA предусмотрено два. Они отличаются типом определения плоскости - через позиции ее нормали или ее 3 точек. Если 
удобно определить плоскость через позиционный вектор ее нормали PN (определяющий и позицию и ориентацию плоскости в пространстве), то функция:

$$
\text { dist }=\operatorname{VdoPn}(\mathbf{A}, \mathbf{P N})=\operatorname{VscalV}(\operatorname{VEd}(\mathbf{P N} \cdot \mathbf{b e}), \operatorname{VmV}(\mathbf{P N} . \mathbf{b}, \mathbf{A}))
$$

возвращает скалярное произведение единичного вектора нормали PN.be (из Poz-вектора $\mathbf{P N}$ ) с вектором разности заданного D3V-вектора точки А и позиции PN.b плоскости (ее нормали). Если же плоскость задана тремя D3V-векторами $\mathbf{V} \mathbf{p}=\left\{\mathbf{V}_{\mathbf{1}}, \mathbf{V}_{\mathbf{2}}, \mathbf{V}_{\mathbf{3}}\right\}$ ее точек, то оператор: $\quad \operatorname{dist}=\operatorname{VdoPv}(\mathbf{A}, \mathbf{V} \mathbf{1}, \mathbf{V} \mathbf{2}, \mathbf{V 3})=$ $=\operatorname{VscalV}(\operatorname{VEd}(\operatorname{VvectV}(\operatorname{VmV}(\mathbf{V} \mathbf{1}, \mathbf{V} \mathbf{2}), \operatorname{VmV}(\mathbf{V 1}, \mathbf{V 3}))), \operatorname{VmV}(\mathbf{V} \mathbf{1}, \mathbf{A}))$

сначала определяет вектор нормали плоскости - в виде векторного произведения разностей вектора ее первой точки $\mathbf{V 1}$ с двумя остальными ее векторами $\mathbf{V 2}$ и V3, после чего он определяет и возвращает искомое расстояние dist от точки А до плоскости 3 точек как скалярное произведение единичного вектора нормали разности заданного D3V-вектора точки и позиции нормали плоскости в виде вектора V1 (очевидно, что может быть выбран любой из Vp).

Определения позиционного, концевого и целевого расстояний Poz-векторов

Операторы PVdoPV, EVdoEV и $\operatorname{doPVPV}(\mathbf{P A}, \mathbf{P B})$ в соответствии с их определениями устроены просто. Они вычисляют модули разностей соответствующих или суммы всех элементов 2 структур двухпозиционных векторов.

Оператор определения кратчайшего расстояния у двух Рoz-векторов

Оператор PVotPV(PA,PB) устроен чуть сложнее. В нем определяется минимум модуля векторов между началами и концами векторов PA.be, PB.be:

$\operatorname{PVotPV}($ PA,PB $)=\min (\operatorname{VMod}(\operatorname{VmV}($ PA.e, PB.e $)), \operatorname{VMod}(\operatorname{VmV}($ PA.b, PB.e $))$, $\operatorname{VMod}(\operatorname{VmV}($ PA.e, PB.b) $)$, $\operatorname{MMod}(\operatorname{VmV}($ PA.b, PB.b) $)$.

Таблица 8 Операции вычисления векторных расстояний

\begin{tabular}{|l|c|}
\hline \multicolumn{1}{|c|}{ Описание } & Oператор \\
\hline \hline Оператор определения расстояния между двумя D3-точками & dist=VdoV(A, B) \\
\hline Оператор определения расстояния D3-точки до плоскости PL & dist =VdoPn|Pv(A,PN|Vp) \\
\hline Определение позиционного расстояния двух Poz-векторов & dist =PVdoPV(PA, PB) \\
\hline Определение концевого расстояния двух Роz-векторов & dist =EVdoEV(PA, PB) \\
\hline Определение целевого расстояния суммы двух Poz-векторов & dist =doPVPV(PA, PB) \\
\hline Определение кратчайшего расстояния у двух Poz-векторов & dist =PVotPV(PA, PB) \\
\hline Определение расстояния скрещивания линий Роz-векторов & dist =PVxPV( PA, PB, D ) \\
\hline Определение расстояния до скрещивания линий Роz-векторов & dist =doPVskrPV(PA, PB, PD) \\
\hline
\end{tabular}

Их устройства весьма очевидны, за исключением последнего оператора табл. 8.

Оператор определения расстояния до скрещивания двух Poz-векторов

Для определения D3-вектора скрещивания D оператору dist=PVxPV не нужно искать точку скрещивания (или пересечения) продолжения их линий. 
Для этого нужно построить $\mathbf{D}^{0}=\operatorname{VEd}(\operatorname{VvectV}(\mathbf{P A} . \mathbf{b e}, \mathbf{P B} . \mathbf{b e}))$ - единичный вектор нормали параллельных плоскостей, содержащих векторы $\mathbf{A}$ и $\mathbf{B},-$ с последующим его скалярным умножением на вектор смещения начал этих векторов:

dist=PVxPV $\left(\mathbf{P A}, \mathbf{P B},{ }^{* \mathbf{D}}\right)=\operatorname{VscalV}\left(\operatorname{VmV}(\mathbf{P A} . \mathbf{b}, \mathbf{P B} . \mathbf{b}), \mathbf{D}^{0}\right) ; \mathbf{D}=\mathrm{VuS}\left(\mathbf{D}^{0}\right.$, dist $)$.

Оператор определения места вектора скрещивания двух Poz-векторов

Важный в робототехнике оператор dist $=\operatorname{doPVxPV}\left(\mathbf{P A}, \mathbf{P B},{ }^{*} \mathbf{P D}\right)$ заключается в определении расстояния $\mathrm{x}=$ dist от начала первого вектора до точки его скрещивания со вторым вектором и построении Poz-вектора PD скрещивания. Вычислимы следущие D3-вектора и синус угла $\sigma$ между исходными векторами:

$\mathbf{A}=\mathbf{P A} . \mathbf{b}, \quad \mathbf{B}=\mathbf{P B} . \mathbf{b}, \quad \mathbf{D}=[\mathbf{A} \times \mathbf{B}], \quad \mathbf{L}=\mathbf{P A} . \mathbf{b}-\mathbf{P B} . \mathbf{b}, \quad \sin \sigma=\left|\left[\mathbf{A}^{\mathbf{0}} \times \mathbf{B}^{\mathbf{0}}\right]\right|$.

В принципе, можно искать алгебраическое решение для двух неизвестных х и у в компонентах векторов А и В в их системе координат, используя систему выбранных двух линейных уравнений из 3 векторных уравнений:

$$
\mathbf{A}^{0} \cdot \mathrm{x}-\mathbf{B}^{0} \cdot \mathrm{y}=\mathbf{L}-\mathbf{D} \text {. }
$$

Но есть и более простое - прямое геометрическое - решение векторных треугольников (рис.10), состоящее из трех пунктов алгоритма в операторах PBA:

1. вектор $\mathbf{C}=\operatorname{Bort} \mathbf{A}(\mathbf{B}, \mathbf{L})$ есть проекция $\mathbf{L}$ на плоскость, ортогональную $\mathbf{B}$;

2. вектор $\mathbf{E}=\operatorname{Bort}\left(\mathbf{D}^{\mathbf{0}}, \mathbf{C}\right)$ есть проекция $\mathbf{A}^{\mathbf{0}} \cdot \mathbf{x}$ на плоскость, ортогональную $\mathbf{B}$;

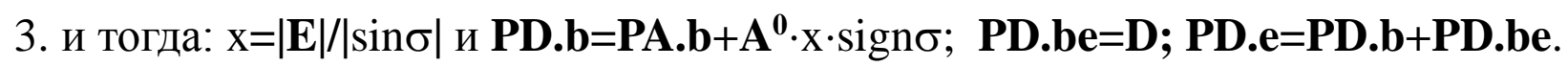

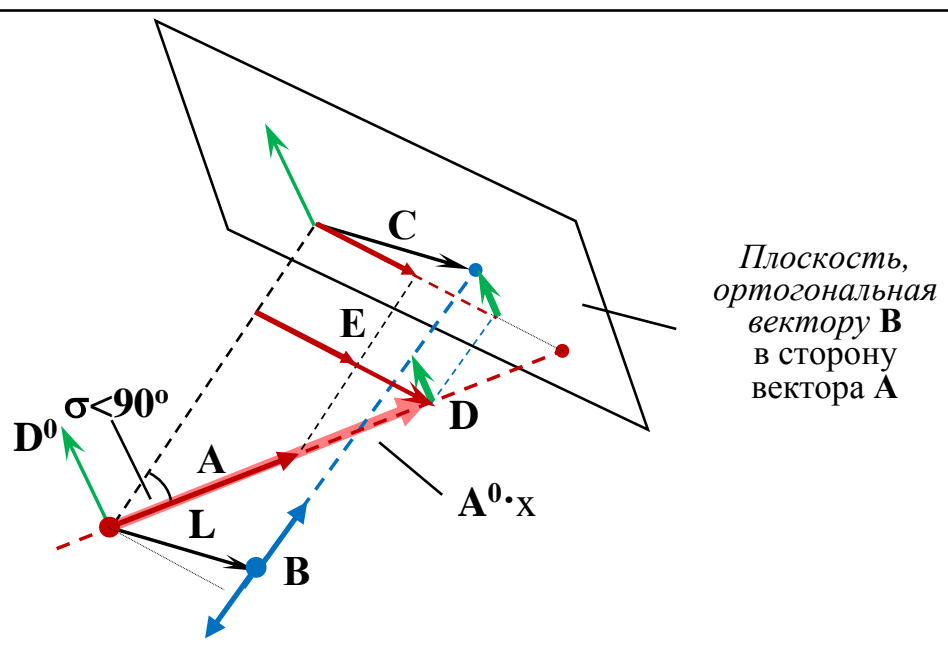

Рис.10. Геометрия задач скрещивающихся векторов. Векторы А и В лежат в параллельных плоскостях

\section{ОПЕРАЦИИ СЕЧЕНИЯ - ПРИКОСНОВЕНИЯ}

В дополнение к операциям ориентации векторов в пространстве в системе PВА имеется важный набор операторов, связанных с позиционированием векторов. К ним относятся операции построения векторов по условиям их точечного прикосновения к геометрическому объекту, его пересечения или линейного совмещения с его границей. Во всех этих случаях операция прикосновения к удаленному объекту является первичной операцией формирования движения. 
Доступным движением может быть не только осевой поворот, но и перемещение в пространстве вдоль некого направления. Границы возможных движений до первичного соприкосновения с объектами окружения определяют размеры свободного или рабочего пространства в пространстве объектов ${ }^{7}$.

Построение операторов прикосновения в системе РВА выполнено исходя из свойства любого протяженного объекта иметь фиксированные точки, позиции которых определяет их положение в пространстве. Так, позиционное описание наиболее значимых векторных объектов - их систем координат - вместе с матрицей направляющих косинусов содержит и вектор точки начала системы координат в базовой системе координат. Позиционный вектор определяется двумя точками, плоскость - тремя, у нелинейных поверхностей число характерных точек растет вместе с числом коэффициентов их уравнений.

Вместе с такими характерными точками, определяющими взаимные позиции объектов, в обратных геометрических задачах системы РВА определяются дополнительные точки прикосновения одного объекта к другому или точки взаимного пересечения двух объектов.

Важным обстоятельством является тот факт, что операция прикосновения, как правило, связана условием меры искомого или фиксированного расстояния до точки прикосновения. Это приводит к необходимости добавления в состав объектов РВА понятия окружности (граница которой формирует условие изометрии расстояния). Соответственно, в операторах РВА появляются операторы прикосновений к окружности и пересечений окружностей (Таблица 9):

Таблица 9

Операции связи векторов с окружностью

\begin{tabular}{|l|c|}
\hline \multicolumn{1}{|c|}{ Описание } & Оператор \\
\hline Оператор построения касательного вектора & $\alpha=\mathrm{VkO}\left(\mathbf{Y}^{0}, \mathbf{P}_{\mathrm{T}}, \mathbf{P}_{\mathrm{C}} \pm \mathrm{R}, \mathbf{V}_{\mathrm{K}}\right)$ \\
\hline Оператор определения векторов пересечения окружностей & $\alpha=\mathrm{OxO}\left(\mathbf{C}_{1}, \mathrm{R}_{1}, \mathbf{C}_{2}, \mathrm{R}_{2}, \mathbf{P}_{1}, \mathbf{P}_{2}\right)$ \\
\hline
\end{tabular}

\section{Построение касательного целевого вектора}

Весьма востребованным в робототехнических приложениях является оператор прикосновение вектора к окружности: $\mathrm{VkO}\left(\mathbf{Y}^{0}, \mathbf{P}_{\mathrm{T}}, \mathbf{P}_{\mathrm{C}}, \pm \mathrm{R}, \mathbf{V}_{\mathrm{K}}\right)$. Необходимость в такой операции связана с существованием обширного класса механизмов с перекрещивающимися осями их звеньев и степеней подвижности. В таких случаях любые повороты звена механизма вокруг перекрещивающейся оси приводят к его «обкатыванию» вокруг окружности с центром в оси враще-

\footnotetext{
${ }^{7}$ Такому пространству объектов интереса до сих пор не найдено удачного имени. В разное время и в разных науках были разные попытки: от Логоса Гераклита, universitas iuris (совокупность вещей) в римском праве, Универсума в Логике до «Террайна» А.А. Кирильченко (среды передвижения с препятствиями). В робототехнике наиболее адекватно существу дела имя «Операционная среда» - связанные с решаемой задачей параметры внешней среды, данные системе управления робота в его сенсорных сигналах (не следует забывать удачные формулы даже опальных философов).
} 
ния, и это нужно учитывать при решении прямых и обратных кинематических задач формирования движения такого механизма.

Содержательно эта операция заключается в построении из заданной точки цели $\mathbf{V}_{\mathrm{T}}$ вектора $\mathbf{V}_{\mathrm{K}}$, касательного к окружности с величиной радиуса $\mathrm{R}$, радиусом-вектором $\mathbf{V}_{\mathrm{C}}$ центра и вектором $\mathbf{Y}^{0}$ нормали её плоскости (рис. 11). Целью операции является угол между вектором $\mathbf{V}_{\mathrm{K}}$ и вектором направления на цель из центра окружности, который зависит от расстояния до цели.

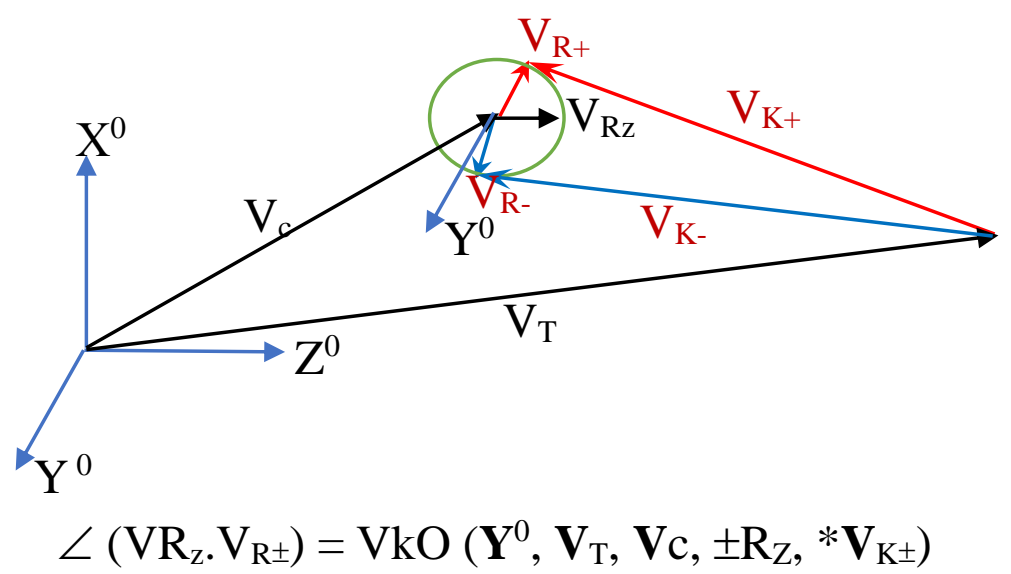

Рис.11. Векторный оператор $\mathrm{VkO}$ для построения касательных векторов из точки $\mathbf{V}_{\mathrm{T}}$ в плоскости $\mathbf{X}^{0} \mathbf{Z}^{0}$, заданной в системе координат окружности векторами центра $\mathbf{V}_{\mathrm{c}}$, оси

$\mathbf{Y}^{0}$ и радиусом R окружности в этой плоскости.

Оператор строит один из двух векторов $\mathbf{V}_{\mathrm{K}}$ и определяет угол между осью $\mathbf{Z}^{0}$ окружности и точкой касания к ней векторов $\mathbf{V}_{\mathrm{K}}$. Векторы $\mathbf{V}_{\mathrm{C}}$ и $\mathbf{V}_{\mathrm{T}}$ предполагаются ортогональными вектору $\mathbf{Y}^{0}$. Выбор правого - левого вектора (со стороны $\mathbf{V}_{\mathrm{T}}$ ) определяется заданным «знаком» величины радиуса окружности $\pm \mathrm{R}_{\mathrm{z}}$.

Очевидно, что у такой задачи имеются два решения - с разных сторон окружности. Поэтому в операторе используется знак у радиуса окружности $\pm \mathrm{R}$. Он определяет выбор соответственно левого или правого решения.

В процедуру этого оператора заложено преобразование ПСК по смыслу задачи: алгоритм задачи описан в векторах системы координат $\mathbf{X}^{0}, \mathbf{Y}^{0}, \mathbf{Z}^{0}$, присоединенной к плоскости рисунка и определенной формальным параметром ее нормали $\mathbf{Y}^{0}$. При этом предполагается, что векторы $\mathbf{V}_{\mathrm{C}}$ и $\mathbf{V}_{\mathrm{T}}$ принадлежат плоскости окружности (ортогональны оси $\mathrm{Y}^{0}$ ), а расстояние от центра окружности до точки $\mathbf{V}_{\text {T }}$ больше радиуса окружности (в противном случае вместо ответа будет отправлен признак нарушения условий задачи - число 999).

Алгоритм этого оператора достаточно прост. Длина и направление искомого вектора касательной $\mathbf{V}_{\mathrm{K}}$ определяются по катету прямоугольного треугольника касания с гипотенузой длины вектора направления на цель из центра окружности (обычно это - центр вертикальной оси поворота манипулятора со схватом, направленным вдоль вектора $\mathbf{V}_{\mathrm{K}}$ ). 


\section{Пересечение окружностей}

Этот весьма нужный оператор $\alpha=\mathrm{OxO}\left(\mathbf{C}_{1}, \mathrm{R}_{1}, \mathbf{C}_{2}, \mathbf{R}_{2},{ }^{*} \mathbf{P}_{1},{ }^{*} \mathbf{P}_{2}\right)$ из множества операторов обратных геометрических задач определяет параметры точек пересечения двух окружностей на плоскости. Он востребован в задачах построения двухзвенных векторов дотягивания до геометрического объекта.

Операция заключается в поиске двух векторов из начала координат в точки $\mathbf{P}_{1}$ и $\mathbf{P}_{2}$ пересечения дуг двух окружностей, заданных векторами их центров $\mathbf{C}_{1}, \mathbf{C}_{2}$ и величинами их радиусов $\mathrm{R}_{1}, \mathrm{R}_{2}$. Важным обстоятельством является то, что окружности строятся в плоскости векторов их центров. Если начало координат совпадает с одним из центров окружностей, то в качестве нормали плоскости окружностей используется орт оси Z рабочей системы координат.

Программа удобна для использования в плоской кинематике дуг и центров звеньев манипулятора. Общий случай пересечения двух конусов, где требуется выравнивание уровней плоскостей их ортогональных сечений с изменением позиций центра и радиуса окружности был рассмотрен выше (см. рис. 7).

Алгебраически решение этой задачи ищется в корнях $\mathbf{P}_{1,2}$ системы двух квадратных уравнений относительно компонент вектора $\mathbf{P}$ :

$$
\left(\mathbf{P}-\mathbf{C}_{1}\right)^{2}=\mathbf{R}_{1}^{2} \text { и }\left(\mathbf{P}-\mathbf{C}_{2}\right)^{2}=\mathbf{R}_{2}^{2} \text {. }
$$

Однако геометрически эта задача решается простым поворотом межцентрового направления вектора радиуса $\mathbf{R}_{10}$ на угол $\alpha=\angle \mathbf{R}_{10} \mathbf{R}_{1}$, определяемый из треугольника $\mathbf{C}_{1}, \mathbf{P}_{\mathbf{1}}, \mathbf{C}_{2}: \quad \mathbf{R}_{10}=\operatorname{VuS}\left(\operatorname{VMod}\left(\operatorname{VEd}\left(\operatorname{VmV}\left(\mathbf{C}_{2}, \mathbf{C}_{1}\right)\right)\right), \mathbf{R}_{1}\right)$;

$$
\begin{gathered}
\mathrm{C}_{12}=\operatorname{VMod}\left(\operatorname{VmV}\left(\mathbf{C}_{2}, \mathbf{C}_{1}\right)\right) ; \\
\cos \alpha=\left(\mathrm{C}_{12}{ }^{2}+\mathrm{R}_{1}{ }^{2}-\mathrm{R}_{2}{ }^{2}\right) /\left(2 \cdot \mathrm{C}_{12} \cdot \mathrm{R}_{1}\right) .
\end{gathered}
$$

Если $\mathrm{C}_{12} \leq\left(\mathrm{R}_{1}+\mathrm{R}_{2}\right)$ и $\mathrm{C}_{12} \geq\left|\mathrm{R}_{1}-\mathrm{R}_{2}\right|-$ окружности пересекаются или касаются. Тогда: $\quad \mathbf{P}_{1}=\mathbf{C}_{1}+\mathrm{R}_{1} \cdot \operatorname{PVV}\left(\mathbf{C}_{12}{ }^{0}, \mathbf{Z}^{0},+\alpha\right)-$ вектор в точку $\mathbf{P}_{1}$, и $\quad \mathbf{P}_{2}=\mathbf{C}_{1}+\mathrm{R}_{1} \cdot \operatorname{PVV}\left(\mathbf{C}_{12}{ }^{0}, \mathbf{Z}^{0},-\alpha\right)-$ вектор в точку $\mathbf{P}_{2}$. Иначе - возвращаются $\alpha=999$ и координаты двух точек $\mathbf{P}_{1}$ и $\mathbf{P}_{2}$ на прямой $\mathbf{C}_{1} \mathbf{C}_{2}$.

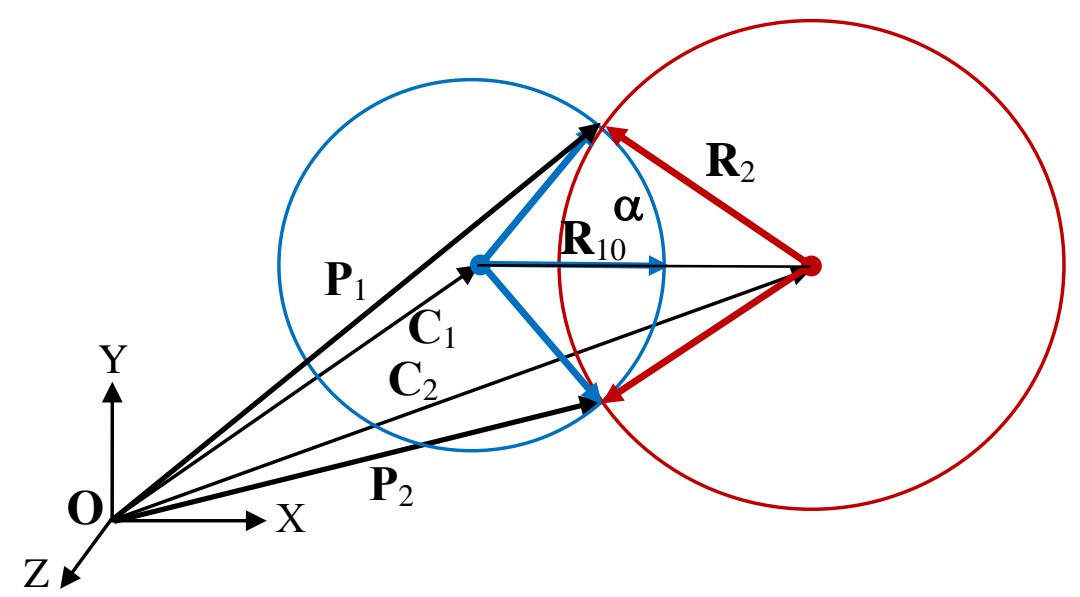

Рис. 12. Векторы пересечения двух окружностей Плоскость окружностей определена тремя точками треугольника $O, C_{1}, C_{2}$ 


\section{ЗАКЛЮЧЕНИЕ}

В эпиграфе к этой работе упомянута мечта об автомате, самостоятельно раскрывающем указанную тему. В системе операторов и программ РВА реализуется подобное спрятанное от автора координатное раскрытие геометрической темы нужного преобразования векторов с исполнением вычислений для семантически описанного взаимодействия векторных объектов.

Отдельные операторы расширения векторной алгебры, такие как PVV и VINT, заменяющие кватернионы и бикватернионы, и другие операторы типа PSK, BortA, VkO и PVK, предназначены для проектирования кинематических схем манипуляторов, движителей роботов, их датчиков и инструмента, для подготовки и контроля бортовых программ, для построения алгоритмов геометрической интерпретации измерений операционных сред и решения задач установочной адаптации с обеспечением требуемой точности программного взаимодействия робота с крупногабаритными изделиями промышленности.

Предложенная семантика методов РВА обеспечивает описание содержания требуемых действий и движений в алфавите операций совмещения позиций объектов в трехмерном пространстве с удобными кинематическими средствами для моделирования расстояний и углов их относительного положения. Обычные векторно-матричные метрические преобразования не нужны в PBA на геометрически понятийном уровне проектирования целенаправленных движений векторных объектов, но, если нужно, они могут быть легко задействованы через обычные скалярные произведения векторных объектов.

Предлагаемая программная реализация системы операторов РВА опробована при решении стандартных кинематических задач построения движений машин. Важно подчеркнуть, что построенный алфавит операторов PBA на множестве задач управления ориентацией КА показал свою эффективность не только как средство быстрого программирования моделей систем ориентации объектов космической техники, но, главное, - как очень эффективный способ геометрического мышления при формировании алгоритмов прямых и обратных геометрических преобразований векторных астросистем любой сложности.

Система PBA полностью открыта для использования и ее развития. Она обеспечивает быстрое алгоритмическое и программное решение прикладных задач формирования целенаправленных движений объектов космической техники и робототехники. Она также является удобным и полезным средством для реализации «интеллектуальных» бортовых программ.

Основное место современного применения операторов РВА - стать средством развития семантики метрических понятий построения движений до уровня семантики геометрических понятий построения поведения машин. 


\section{ЛИТЕРАТУРА}

(в хронологическом порядке)

1. Ефремов Д. Новая геометрия треугольника. Одесса, 1902 г. // Электронное издание: URL: http://www.math.ru/lib/book/djvu/ngt/ngt.djvu

2. Платонов А.К. Исследование свойств корректирующих маневров межпланетных полетов//Космические исследования т.4, вып.5,1966. С.670-693.

3. Казакова Р.К., Платонов А.К. Язык для описания вращения космического аппарата. //В сб. "Управление в пространстве". М.: Наука, Т.1, 1973, С. 52-63. // Препринты ИПМ им. М.В. Келдыша, 1971, № 59, 34c.

4. Бранец В.Н., Шмыглевский И.П. Применение кватернионов в задачах ориентации твёрдого тела. М.: Наука, 1973, 320 с..

5. Кантор И.Л., Солодовников А.С. Гиперкомплексные числа // М.: Наука, 1973 г., 144 с.

6. Попов Е.П., Верещагин А.Ф., Зенкевич С.Л. Манипуляционные роботы: динамика и алгоритмы. М.: Наука, 1980.

7. Притыкин Ф.Н. Параметрический метод моделирования огибающих поверхностей применительно к движению манипулятора в среде с препятствиями. Кандидатская диссертация. М.: МАИ. 1987. - 194 с.

URL:http://www.dissercat.com/content/geometricheskoe-issledovanie-i-sintezmalykh-dvizhenii-mobilnykh-i-statsionarnykh-robotov-v-

8. Платонов А.К. Геометрические преобразования в робототехнике // Новое в жизни, науке, технике. Сер. "Математика, кибернетика"; № 4.

М.: Знание (). 1988г. С. 32.

9. Каляев А.В., Чернухин Ю.В., Носков В.Н., Каляев И.А. Однородные управляющие структуры адаптивных роботов. М.: Наука, 1990, 152 с.

10. Препарата Ф., Шеймос М. Вычислительная геометрия: Введение. Пер. с англ. М.: Мир, 1989, 478 с.

11. Платонов А.К. Метод определения кинематических характеристик роботов // "Программирование прикладных систем". М.: Наука, 1992, С. 181-189.

12. Комаров М.М. Система геометрического моделирования и её применение. // "Программирование прикладных систем". М.: Наука, 1992, С. 189-196.

13. Белоусов И.Р., Карташов В.А. Натурное моделирование движений манипулятора в реальном масштабе времени. //"Программирование прикладных систем". М.: Наука, 1992, С. 214-219.

14. Платонов А.К., Казакова Р.К., Громова Л.Т. Разработка методов оценки требований к точности изготовления и паспортизации промышленных роботов. // "Программирование прикладных систем". М.: Наука, 1992, С. 248-261. 
15. Притыкин Ф.Н. Геометрическое моделирование при решении задач робототехники: учеб. Пособие. Омск: ОмГТУ, 1998. - 71 с.

16. Голубев Ю.Ф. Основы теоретической механики. М.: Издательство МГУ. 2000. $719 \mathrm{c}$.

17. Белоусов И.Р. Формирование уравнений динамики роботов-манипуляторов // Препринты ИПМ им. М.В. Келдыша. 2002. № 45.

18. Изыскания и проектирование железных дорог. - М.: ИКЦ «Академкнига», 2003. - 288 c.

19. Зенкевич С.Л., Ющенко А.С. Основы управления манипуляционными роботами: учеб. для вузов. 2-е изд., перераб. и доп. М.: Изд-во МГТУ им. Н.Э. Баумана, 2004. 480 с.

20. Челноков Ю.Н. Кватернионные и бикватернионные модели и методы механики твердого тела и их приложения. Геометрия движения. Саратов: Изд-во Саратовского университета, 2006. 236 с.

21. Гордеев В.Н. Кватернионы и трехмерная геометрия. Киев. 2012. 60 с.

22. Балакин П.Д., Шамутдинов А.Х. Схемное решение механизма пространственного манипулятора // Омский научный вестник. 2012.

23. Голубев Ю.Ф. Алгебра кватернионов в кинематике твердого тела // Препринты ИПМ им. М.В. Келдыша. 2013. № 39, 23 с.

URL: http://library.keldysh.ru/preprint/asp?id=2013-39

24. Челноков Ю. Н. Бикватернионное решение кинематической задачи управления движением твердого тела и его приложение к решению обратных задач кинематики роботов-манипуляторов // Изв. РАН. Механика твердого тела. 2013. № 1. C. 38-58.

25. Шиманчук Д.В. Теоретическая механика. Кинематика твердого тела. СПб.: СПбГУ. 2013.

http://www.apmath.spbu.ru/ru/staff/shimanchuk/files/kinematics_body1.pdf

26. Уроки Flash (Инверсная кинематика в 3D Studio Max) //

URL: http://www.lessonsflash.ru/3ds/Index.html.

27. Дыда А.А., Оськин Д.А. Решение обратной задачи кинематики для манипуляционного робота методом штрафных функций // Фундаментальные исследования. - 2015. - № 11-4. - С. 673-677;

URL: http://www.fundamental-research.ru/ru/article/view?id=39485

28. Simulated Reference Platform Microsoft Robotics Developer Studio 


\section{Оглавление}

ВВЕДЕНИЕ 3

ГЕОМЕТРИЧЕСКОЕ МЫШЛЕНИЕ И КООРДИНАТНАЯ РЕАЛИЗАЦИЯ .............................6

ВИДЫ ИСПОЛЬЗУЕМЫХ ДАННЫХ ..............................................................................9

ПРИСОЕДИНЁННАЯ СИСТЕМА КООРДИНАТ И ОРТОГОНАЛИЗАЦИЯ ВЕКТОРОВ ...11

Ортогонализация и проецирование векторов репера ........................................................13

ВЕКТОРНЫЕ АЛГОРИТМЫ КОНИЧЕСКИХ ПОВОРОТОВ ..................................................15

Базовый оператор поворота вектора вокруг вектора ....................................................15

Операторы построения нужных конических движений .....................................................17

Отображение вектора на конус ............................................................ 18

Угол поворота вектора под вектор ...................................................... 18

Поворот вектора до совмещчения с плоскостью ......................................... 19

Построение векторов на конусе под углом к другому вектору.................... 19

Определение оси и угла Эйлерового поворота пары векторов ....................................20

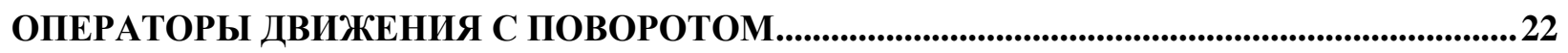

Операторы плоских транспортных движений ...................................................................23

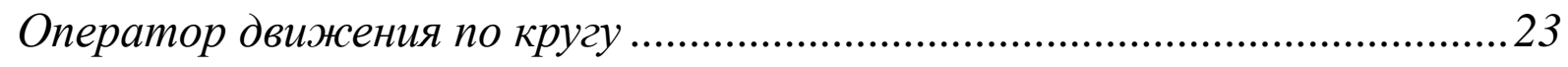

Оператор рельсового поворота.......................................................22

Оператор формирования гладких траекторий ........................................... 24

Оператор вальсирующуего движения .........................................................24

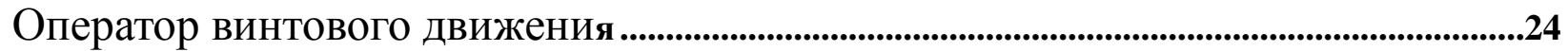

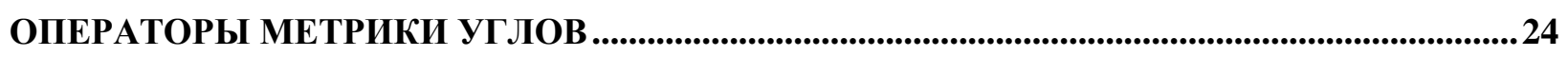

Определение угла между векторами....................................................26

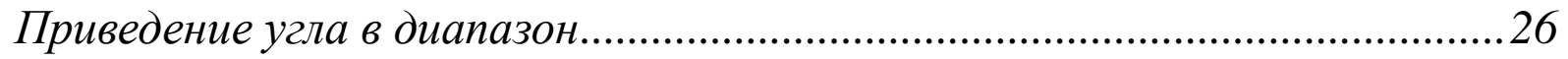

Операция "Угль треугольника" ................................................................... 26

Определение угла вектора с конусом ........................................................2 26

ОПЕРАТОРЫ МЕТРИКИ РАССТОЯНИЙ ...........................................................................................2 27

Оператор определения расстояния между векторными D3V-точками ....27

Oператор определения расстояния D3V-точки до плоскости PL .............. 27

Определения концевого и цуелевого расстояний Роz-векторов......................228

Оператор определения кратчайшего расстояния у двух Роz-векторов..... 28

Оператор определения расстояния до скрещчивания двух Роz-векторов ... 28

Оператор определения места вектора скрещивания двух Роz-векторов .. 29 ОПЕРАЦИИ СЕЧЕНИЯ - ПРИКОСНОВЕНИЯ .29

Построение касательного целевого вектора....................................................................................30

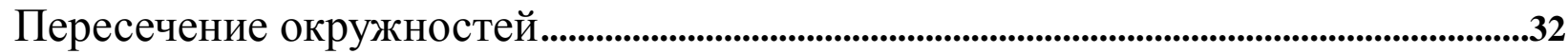

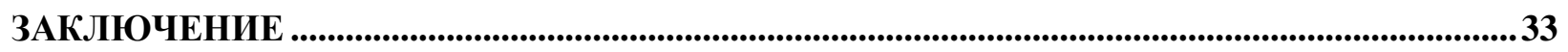

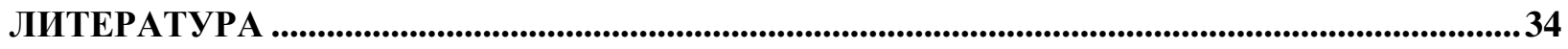

